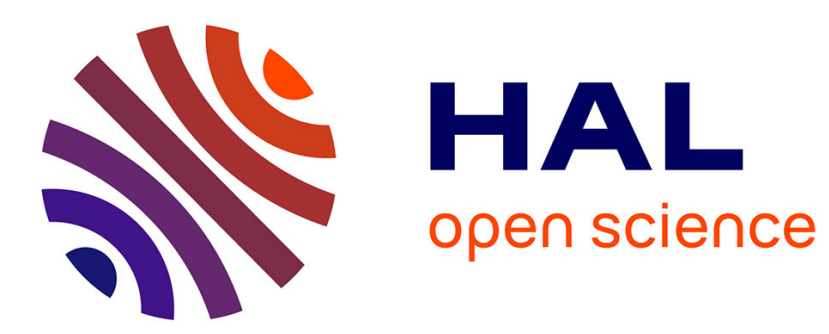

\title{
Boundary Value Problems of Elasticity with Unilateral Constraints
}

\author{
Gaetano Fichera
}

\section{To cite this version:}

Gaetano Fichera. Boundary Value Problems of Elasticity with Unilateral Constraints. Linear Theories of Elasticity and Thermoelasticity, Springer Berlin Heidelberg, pp.391-424, 1973, 10.1007/978-3-66239776-3_4. hal-02183016

\section{HAL Id: hal-02183016 https://hal.science/hal-02183016}

Submitted on 14 Jul 2019

HAL is a multi-disciplinary open access archive for the deposit and dissemination of scientific research documents, whether they are published or not. The documents may come from teaching and research institutions in France or abroad, or from public or private research centers.
L'archive ouverte pluridisciplinaire HAL, est destinée au dépôt et à la diffusion de documents scientifiques de niveau recherche, publiés ou non, émanant des établissements d'enseignement et de recherche français ou étrangers, des laboratoires publics ou privés. 


\section{Boundary Value Problems of Elasticity with Unilateral Constraints.}

\section{Gaetano Fichera.}

In the preceding article "Existence Theorems in Elasticity", which henceforth will be cited as E.T.E. ${ }^{1}$. I have treated boundary value problems of Elasticity in the case when the side conditions to be associated with the differential equations of equilibrium correspond to bilateral constraints imposed upon the elastic body. In this article I will treat the analytical problems which arise when unilateral constraints are imposed. In Sect. 6 of E.T.E. it is shown that the "bilateral problems", as far as the existence theory is concerned, are founded on the solution of a system of equations of the following type

$$
B(u, v)=F(v), \quad u \in V, \forall v \in V,
$$

where $B(u, v)$ is a bounded bilinear form defined in $H \times H(H=$ Hilbert space $)$, $F$ is a linear functional and $V$ is a closed linear subspace of $H$. These equations, in the case when $B$ is symmetric and the space $H$ real, are easily obtained by imposing upon the energy functional

$$
\mathscr{I}(v)=\frac{1}{2} B(v, v)-F(v)
$$

the condition of attaining a minimum on $V$.

In the case of unilateral constraints, the manifold $V$ is not a closed linear subspace of $H$ but a closed convex set of $H$. The condition for $\mathscr{I}(v)$ to have a minimum in the point $u$ of $V$ leads, in this case, to the inequalities

$$
B(u, v-u) \geqq F(v-u), \quad u \in V, \forall v \in V
$$

which easily reduce to (1) if $V$ is a linear subspace of $H$.

In order to provide, as in the case of bilateral constraints, satisfactory foundations for unilateral problems of elasticity, we shall develop in the first two sections of this article an abstract theory of functional inequalities (2), considering not only the case of a symmetric bilinear form $B(u, v)$ but also the non symmetric case. We shall denote these problems as abstract unilateral problems.

1. Abstract unilateral problems: the symmetric case. Let $H$ be a real Hilbert space. We denote by $(u, v)$ the scalar product in $H$ and by $\|u\|$ the norm of the vector $u$ of $H$. Let $B(u, v)$ be a symmetric bounded bilinear form defined on $H \times H$. From the elementary theory of Hilbert spaces it is well known that there exists one and only one symmetric, bounded linear operator $T$ from $H$ into $H$ such that $B(u, v)=(T u, v)$ for any $u$ and $v$ of $H$. Let $N(T)$ be the kernel of the operator $T$, i.e.

$$
N(T) \equiv\{v ; T(v)=0\} .
$$

\footnotetext{
1 See this volume, pp. 347-389.
} 
The linear subspace $N(T)$ is also the kernel of the nonnegative quadratic form $B(v, v)$, i.e.

$$
N(T) \equiv\{v ; B(v, v)=0\} .
$$

Let $Q$ be the orthogonal projector of $H$ into the kernel of $B(v, v)$ and set $P=I-Q$ ( $I=$ identity operator). We shall assume the following hypotheses.

(I) Semi-coerciveness hypothesis:

$$
B(v, v) \geqq c\|P v\|^{2} \quad \forall v \in H,
$$

where $c$ is a positive constant independent on $v$.

(II) The kernel of the quadratic form $B(v, v)$ is finite dimensional.

Let $F(v)$ be a bounded linear functional defined on $H$ and $V$ a closed convex set of $H .^{2}$

\section{I Let us consider the functional}

$$
\mathscr{I}(v)=\frac{1}{2} B(v, v)-F(v) .
$$

There exists in $V$ a vector $u$ which minimizes $\mathscr{I}(v)$ in $V$, if and only if there exists a solution of the unilateral problem.

$$
B(u, v-u) \geqq F(v-u), \quad u \in V, \forall v \in V .
$$

If $\mathscr{I}(v)$ has a minimum for $v=u$, given any $v \in V$, the function of $t(0 \leqq t \leqq 1)$ $g(t)=\mathscr{I}[u+t(v-u)]$ has a minimum for $t=0$. The condition $g^{\prime}(0) \geqq 0$ exactly coincides with (1.2). Conversely if (1.2) is satisfied for any $v \in V$, we have

$\mathscr{I}(v)-\mathscr{I}(u)=\mathscr{I}[u+(v-u)]-\mathscr{I}(u)=B(u, v-u)-F(v-u)+\frac{1}{2} B(v-u, v-u) \geqq 0$.

Remark that in the proof of this lemma only the condition $B(v, v) \geqq 0$ has been used.

Let $U$ be a point set of $H$ containing some $u \neq 0$. Let us consider for any $u \leqslant U, u \neq 0$, the set of nonnegative numbers $t$ such that $t\|u\|^{-1} u$ is contained in $U$. We shall denote by $p(u, U)$ the supremum of this set, i.e.

$$
p(u, U)=\sup \left\{t ; u \in U, u \neq 0, t\|u\|^{-1} u \in U\right\} .
$$

If $T$ is a mapping from $H$ into $H$ and $U$ a set of $H$, by $T[U]$ we shall indicate the image of $U$ under the mapping $T$.

Let $N(F)$ be the kernel of the functional $F$, i.e.

Set

$$
N(F)=\{v ; F(v)=0\} .
$$

$$
\begin{aligned}
L & =N(F) \cap N(T), \\
N(T) & =L \oplus L_{\mathbf{1}} .
\end{aligned}
$$

Let $\widetilde{Q}$ be the orthogonal projector of $H$ onto $L$. Set $\widetilde{P}=I-\widetilde{Q}$. Let $Q_{1}$, be the orthogonal projector of $H$ onto $L_{\mathbf{1}}$.

1.II The functional $\mathscr{I}(v)$ has an absolute minimum in $V$ if there exists $u_{0} \in V$ such that the following conditions are satisfied

(i) $F(\varrho)<0$ for $\varrho \in N(T) \cap V\left(u_{0}\right), p\left\{Q_{1} \varrho, Q_{1}\left[N(T) \cap V\left(u_{0}\right)\right]\right\}=+\infty$.

(ii) The set $\widetilde{P}\left[V\left(u_{0}\right)\right]$ is closed.

2 When we say that $V$ is a convex set, we mean that $v_{1} \in V, v_{2} \in V$ imply $t_{1} v_{1}+t_{2} v_{2} \in V$ for any $t_{1}, t_{2}$ such that $0 \leq t_{i} \leq 1(i=1,2), t_{1}+t_{2}=1$. By $V\left(u_{0}\right)$ we denote the set of all $v$ such that $v+u_{0} \in V$. 
Set $Q=I-P$. Since $L$ is a subspace of $N(T)$, we have $\widetilde{Q}<Q$ and therefore $\widetilde{P}>P$. Let $i$ be the infimum (finite or not) of $\mathscr{I}(v)$ in $V$.

Let $\left\{u_{n}\right\}$ be a minimizing sequence for $\mathscr{I}(v)$ in $V$, i.e.

$$
\lim _{n \rightarrow \infty} \mathscr{I}\left(u_{n}\right)=i, \quad u_{n} \in V .
$$

Set $z_{n}=u_{n}-u_{0}$. The sequence $\left\{z_{n}\right\}$ is minimizing for the functional $\mathscr{I}\left(w+u_{0}\right)$ for $w \in V\left(u_{0}\right)$. Suppose we have proved that $\left\{\widetilde{P} z_{n}\right\}$ contains a bounded subsequence. Then we may extract a subsequence, which we still denote by $\left\{z_{n}\right\}$, such that $\left\{\widetilde{P} z_{n}\right\}$ converges weakly to some limit. This limit must belong to $\widetilde{P}\left[V\left(u_{0}\right)\right]$, since this set is closed and convex and, in consequence, weakly closed. Let $\widetilde{P} z$ be the weak limit of $\left\{\widetilde{P} z_{n}\right\}$. Since $F(\widetilde{Q} v)=0$, we have

$$
\mathscr{I}\left(z_{n}+u_{0}\right)=\mathscr{I}\left(\widetilde{P} z_{n}+u_{0}\right) .
$$

On the other hand, since $B(v, v)$ is lower semicontinuous with respect to weak convergence $^{3}$ and we may suppose $z \in V\left(u_{0}\right)$, we have

$$
i=\lim _{n \rightarrow \infty} \mathscr{I}\left(\widetilde{P} z_{n}+u_{0}\right) \geqq \mathscr{I}\left(\widetilde{P} z+u_{0}\right)=\mathscr{I}\left(z+u_{0}\right) \geqq i .
$$

Assuming $u=z+u_{0}$, we have $u \in V$ and $\mathscr{I}(u)=i$. Thus we have to prove that, from the sequence $\left\{\widetilde{P} z_{n}\right\}$, we may extract a bounded subsequence. Set $\sigma_{n}=\left\|\widetilde{P} z_{n}\right\|$ and suppose $\lim _{n \rightarrow \infty} \sigma_{n}=+\infty$. We have, using (1.1)

$$
c\left\|P z_{n}\right\|^{2} \leqq B\left(z_{n}, z_{n}\right)=2 \mathscr{I}\left(u_{n}\right)-2 \mathscr{I}\left(u_{0}\right)+2 F\left(z_{n}\right)-2 B\left(u_{0}, z_{n}\right) .
$$

Set $w_{n}=\sigma_{n}^{-1} z_{n}$. We have, denoting by $c_{1}$ a suitable constant,

$$
c \sigma_{n}^{2}\left\|P w_{n}\right\|^{2} \leqq c_{1}+2\left(\|F\|+\|T\|\left\|u_{0}\right\|\right) \sigma_{n}\left\|\widetilde{P} w_{n}\right\|
$$

which implies $\lim _{n \rightarrow \infty}\left\|P w_{n}\right\|=0$. We have $Q_{1}=\widetilde{P}-P . Q_{1}$ is a projector which is orthogonal to $P$. Hence

$$
\left\|\widetilde{P} w_{n}\right\|^{2}=\left\|P w_{n}\right\|^{2}+\left\|Q_{1} w_{n}\right\|^{2}=1 .
$$

Since $Q_{1} w_{n} \in N(T)$, we may extract (because of Hypothesis (II)) from $\left\{w_{n}\right\}$ a subsequence, which we still denote by $\left\{w_{n}\right\}$, such that $\left\{Q_{1} w_{n}\right\}$ converges (strongly) to some vector $\varrho$ belonging to $Q_{1}[N(T)]$ and such that $\|\varrho\|=1$. We have $z_{n} \in V\left(u_{0}\right)$. Moreover $\{0\} \in V\left(u_{0}\right)$. Hence, for any $t>0$ and $n$ large enough, $t w_{n} \in V\left(u_{0}\right)$. Then $t \widetilde{P} w_{n} \in \widetilde{P}\left[V\left(u_{0}\right)\right]$ and, for condition ii),

$$
t \varrho=\lim _{n \rightarrow \infty} t \widetilde{P} w_{n} \in \widetilde{P}\left[V\left(u_{0}\right)\right] .
$$

Let $\varrho^{\prime}$ be a vector of $V\left(u_{0}\right)$ such that $\widetilde{P} \varrho^{\prime}=\varrho$. We have $\varrho^{\prime}=\varrho+\left(\varrho^{\prime}-\varrho\right)$, $\varrho \in Q_{1}[N(T)], \varrho^{\prime}-\varrho \in \widetilde{Q}[N(T)]$; thus $\varrho^{\prime} \in N(T) \cap V\left(u_{0}\right)$ and $F(\varrho)=F(\varrho)+F\left(\varrho^{\prime}-\varrho\right)=$ $F\left(\varrho^{\prime}\right)<0$.

3 Suppose that $B(u, v)$ is a symmetric bounded bilinear form and $B(v, v) \geqq 0$. Suppose that $\left\{w_{n}\right\}$ converges weakly to the limit $w$. We have $B\left(w_{n}, w_{n}\right)=B(w, w)+2 B\left(w, w_{n}-w\right)+$ $B\left(w_{n}-w, w_{n}-w\right)$. Since $B\left(w_{n}-w, w_{n}-w\right) \geqq 0, \lim _{n \rightarrow \infty} B\left(w_{n}, w_{n}-w\right)=0$, then

$$
\operatorname{minlim}_{n \rightarrow \infty} B\left(w_{n}, w_{n}\right) \geqq B(w, w) .
$$

Of course the hypothesis of the symmetry of $B(u, v)$ is not restrictive. In fact, if $B(u, v)$ is not symmetric, we can apply the argument to $\frac{1}{2}\{B(u, v)+B(v, u)\}$. 
From (1.3) we deduce the inequality

$$
c \sigma_{n}\left\|P w_{n}\right\|^{2} \leqq \frac{2}{\sigma_{n}}\left[\mathscr{I}\left(u_{n}\right)-\mathscr{I}\left(u_{0}\right)\right]+2 F\left(P w_{n}\right)+2 F\left(Q_{1} w_{n}\right)-2 B\left(u_{0}, P w_{n}\right),
$$

which is absurd since the minlim of the left hand side is nonnegative while the max lim of the right hand side is negative.

Remark. If the set $N(T) \cap V\left(u_{0}\right)$ is bounded [and ii) holds], the existence of the minimum holds for any given $F$. In particular this condition is satisfied if $V$ is bounded. In this case the condition (1.1) is not needed, but only $B(v, v) \geqq 0$. In fact, if $V$ is bounded, any minimizing sequence is bounded and, in consequence, weakly compact.

1.III If $\mathscr{I}(v)$ has a minimum in $V$, for any $u_{0} \in V$ and any $\varrho$ such that

$$
\varrho \in N(T) \cap V\left(u_{0}\right), \quad p\left\{Q_{1} \varrho, Q_{1}\left[N(T) \cap V\left(u_{0}\right)\right]\right\}=+\infty
$$

one must have $F(\varrho)<0$.

Let $\varrho(t)$ be such that $Q_{1} \varrho(t)=t Q_{1} \varrho, \varrho(t) \in N(T) \cap V\left(u_{0}\right) t>0$. From (1.2) we have: $B\left(u, \varrho(t)+u_{0}-u\right) \geqq F\left[\varrho(t)+u_{0}-u\right]$. Since $F[\varrho(t)]=t F(\varrho) \neq 0$, we have $t F(\varrho) \leqq B\left(u, u_{0}\right)-B(u, u)+F\left(u-u_{0}\right)$, which implies $F(\varrho)<0$.

This theorem proves that condition i) of Theorem 1.III is necessary for the existence of the minimum. Let us prove, by an example, that, if condition ii) does not hold, the theorem could fail to be true.

Let $H$ be a two dimensional space, and let $v_{1}$ and $v_{2}$ be two orthogonal unit vectors of $H$. Assume $B=\left(u, v_{1}\right)\left(v, v_{1}\right)$. Let $V$ be the convex set defined by the conditions

Assume that

$$
0 \leqq\left(v, v_{1}\right)<1 \quad\left(v, v_{2}\right)\left[1-\left(v, v_{1}\right)\right] \geqq\left(v, v_{1}\right) .
$$

$$
F(v)=a\left(v, v_{1}\right)+b\left(v, v_{2}\right)
$$

with $a \geqq 1$. The kernel $N(T)$ is defined by the condition $\left(v, v_{1}\right)=0$. For any choice of $u_{0}$ the set $N(T) \cap V\left(u_{0}\right)$ is formed by the vectors $\varrho$ such that $\left(\varrho, v_{1}\right)=0$, $\left(\varrho, v_{2}\right) \geqq \sigma$, where $\sigma$ is a constant depending on $u_{0}$. If $b \neq 0$ the condition $F(\varrho)<0$ can be satisfied only if we assume

$$
\left(u_{0}, v_{2}\right)\left[1-\left(u_{0}, v_{1}\right)\right]=\left(u_{0}, v_{1}\right) .
$$

In this case $F(\varrho)<0$ is equivalent to $b<0$. In the case when $b<0$ the space $L$ coincides with $\{0\}$, and the functional $\mathscr{I}(v)$ has a minimum in $V$, as is easily checked by elementary arguments. If $b=0$, the space $L_{M}$ is the space $\left(v, v_{1}\right)=0$. Then the set $\widetilde{P}\left[V\left(u_{0}\right)\right]$ is defined by the conditions

$$
\left(v, v_{2}\right)=0, \quad-\alpha^{2} \leqq\left(v, v_{1}\right)<1-\alpha^{2}
$$

and it is not closed, i.e. condition ii) of Theorem 1.III is violated. It is easily seen that in this case $\mathscr{I}(v)$ has no minimum in $V$.

1.IV If for any $w \in P[H]$ there exists a $\varrho \in N(T)$ such that $w+\varrho \in V$, and if $F(\varrho)=0$ for every $\varrho \in N(T)$, then $\mathscr{I}(v)$ has a minimum in $V$ and the vector $u$ which minimizes $\mathscr{I}(v)$ in $V$ minimizes $\mathscr{I}(v)$ in the whole $H$.

The existence of the vectors $u$ and $u_{0}$ minimizing $\mathscr{I}(v)$ respectively in $V$ and in $H$, follows from Theorem 1.II.

Let $\varrho \in N(T)$ and $P u_{0}+\varrho \in V$. We have

hence $\mathscr{I}(u)=\mathscr{I}\left(u_{0}\right)$.

$$
\mathscr{I}\left(u_{0}\right)=\mathscr{I}\left(P u_{0}\right)=\mathscr{I}\left(P u_{0}+\varrho\right) \geqq \mathscr{I}(u) \text {; }
$$


$1 . \mathrm{V}$ If $u$ minimizes $\mathscr{I}(v)$ in $V$, the vector $P u$ is uniquely determined by $F$. Any other vector $u^{\prime}$ minimizing $\mathscr{I}(v)$ in $V$ is given by $u^{\prime}=u+\varrho$, where $\varrho$ is a vector of $N(T)$ such that $F(\varrho)=0, u+\varrho \in V$.

Let $u$ be a solution of the unilateral problem (1.2), i.e. a vector minimizing $\mathscr{I}(v)$ in $V$. Let $u^{\prime}$ be a solution of the unilateral problem

$$
B\left(u^{\prime}, v-u^{\prime}\right) \geqq F^{\prime}\left(v-u^{\prime}\right), \quad u^{\prime} \in V, \forall v \in V .
$$

$F^{\prime}$ is a linear bounded functional. From (1.2), (1.4) we deduce

hence

$$
\begin{gathered}
B\left(u, u^{\prime}-u\right) \geqq F\left(u^{\prime}-u\right) \\
B\left(u^{\prime}, u-u^{\prime}\right) \geqq F^{\prime}\left(u-u^{\prime}\right) ;
\end{gathered}
$$

and, by (1.1),

$$
B\left(u-u^{\prime}, u-u^{\prime}\right) \leqq F\left(u-u^{\prime}\right)-F^{\prime}\left(u-u^{\prime}\right)
$$

$$
c\left\|P u-P u^{\prime}\right\|^{2} \leqq\left\|F-F^{\prime}\right\|\left\|u-u^{\prime}\right\|,
$$

which, for $F=F^{\prime}$, implies $P u=P u^{\prime}$. If $u^{\prime}$ minimizes $\mathscr{I}(v)$ in $V$, one has $P u=P u^{\prime}$ and $u^{\prime}=u+\varrho$. Since $\mathscr{I}\left(u^{\prime}\right)=\mathscr{I}(u)-F(\varrho)$, it follows that $F(\varrho)=0$. Conversely, if one has $u^{\prime}=u+\varrho$ and $\varrho$ satisfies the conditions stated in the theorem, $u^{\prime}$ minimizes $\mathscr{I}(v)$ in $V$.

2. Abstract unilateral problems: the nonsymmetric case. We maintain in this section all the hypotheses stated for $B(u, v)$, for $V$ and for $F$, in Sect. 1, but we no longer assume $B(u, v)$ to be symmetric. As in Sect. 1 we denote by $Q$ the orthogonal projector of $H$ onto the kernel of the quadratic form $B(v, v)$ and set $P=I-Q$. Thus $B(u, v)$ is a bounded bilinear form defined on $H \times H$ and satisfying Hypotheses I and II of Sect. 1. $V$ is a closed convex set of $H$. $F$ is a bounded linear functional defined in $H$.

We have $B(u, v)=(T u, v)$ where $T$ is a bounded linear operator from $H$ into $H$. We shall denote by $T^{*}$ the adjoint operator of $T$, i.e. the bounded linear operator defined by the condition

We have

$$
(T u, v)=\left(u, T^{*} v\right), \quad \forall u, v \in H .
$$

$$
B(v, v)=(T v, v)=\left(T^{*} v, v\right)=\frac{1}{2}\left(\left(T+T^{*}\right) v, v\right) .
$$

Since $B(v, v)$ is nonnegative, its kernel coincides with the kernel $N\left(T+T^{*}\right)$ of the symmetric operator $T+T^{*}$, and moreover

$$
N(T) \equiv N\left(T^{*}\right)<N\left(T+T^{*}\right) .
$$

We shall consider the unilateral problem (1.1) under these more general hypotheses on $B$.

Let us denote by $K(T)$ the orthogonal complement of $N(T)$ with respect to $N\left(T+T^{*}\right)$, i.e.

$$
N\left(T+T^{*}\right)=N(T) \oplus K(T) .
$$

The following theorem generalizes Theorem 1.III to a nonsymmetric form $B(u, v)$. As in Sect. 1 we set

$$
L=N(F) \cap N(T), \quad N(T)=L \oplus L_{1},
$$

$\widetilde{Q}, \widetilde{P}, Q_{1}$ have the same meaning as in Sect. 1 . 


\section{I The unilateral problem}

$$
B(u, v-u) \geqq F(v-u), \quad u \in V, \forall v \in V
$$

for the (not necessarily symmetric) bilinear form $B(u, v)$ has a solution $u$ if there exists a $u_{0} \in V$ such that the following conditions are satisfied:

(i) $F(\varrho)<0$ for $\varrho \in N(T) \cap V\left(u_{0}\right), p\left\{Q_{1} \varrho, Q_{1}\left[N(T) \cap V\left(u_{0}\right)\right]\right\}=+\infty$.

(ii) The set $\widetilde{P}\left[V\left(u_{0}\right)\right]$ is closed.

(iii) Let $Q_{0}$ be the orthogonal projector of $H$ onto $K(T)$. For every $\varrho$ satisfying the conditions $Q_{0} \varrho \neq 0, \varrho \in N\left(T+T^{*}\right) \cap V\left(u_{0}\right), p\left\{\left(Q_{0}+Q_{1}\right) \varrho,\left(Q_{0}+Q_{1}\right)\left[N\left(T+T^{*}\right)\right.\right.$ $\left.\left.\cap V\left(u_{0}\right)\right]\right\}=+\infty$ there exists a vector $v_{\varrho} \in V$ such that

$$
F(\varrho)+B\left(\varrho, v_{\varrho}\right)<0 .
$$

Let $f$ be a vector of $H$ such that

$$
F(v)=(f, v), \quad \forall v \in H .
$$

Let us consider the unilateral problem

$$
(u, w-u) \geqq(f, w-u), \quad u \in \widetilde{P}\left[V\left(u_{0}\right)\right], \forall w \in \widetilde{P}\left[V\left(u_{0}\right)\right] .
$$

This is a particular case of the problem solved by Theorem 1.II, when we assume $B(u, v)=(u, v)$, as Hilbert space the space $\widetilde{P}[H]$ and as convex set $\widetilde{P}\left[V\left(u_{0}\right)\right]$. Since in this case the kernel of the quadratic form is $\{0\}$, we have a solution for any given $f$. This solution is unique and, in this particular case, (1.5) gives

$$
\|u-x\| \leqq\|f-g\| \text {; }
$$

$x$ is the solution of the unilateral problem

$$
(x, w-x) \geqq(g, w-x), \quad x \in \widetilde{P}\left[V\left(u_{0}\right)\right], \forall w \in \widetilde{P}\left[V\left(u_{0}\right)\right] .
$$

Let us denote by $R f$ the solution $u$ of the unilateral problem (2.2).

For every positive integer $n$ set $T_{n}=T+n^{-1} I$. Consider the unilateral problem

$$
\left(T_{n} \zeta, w-\zeta\right) \geqq\left(f-T u_{0}, w-\zeta\right), \quad \zeta \in \widetilde{P}\left[V\left(u_{0}\right)\right], \forall w \in \widetilde{P}\left[V\left(u_{0}\right)\right] .
$$

Let $\lambda$ be a positive constant. We can write (2.4) as follows

$$
(\zeta, w-\zeta) \geqq\left(\zeta-\lambda T_{n} \zeta+\lambda f-\lambda T u_{0}, w-\zeta\right), \quad \zeta \in \widetilde{P}\left[V\left(u_{0}\right)\right], \forall w \in \widetilde{P}\left[V\left(u_{0}\right)\right] .
$$

Then a solution of (2.4) exists if and only if there exists a solution $\zeta$ of the equation

$$
\zeta=R\left(\zeta-\lambda T_{n} \zeta+\lambda f-\lambda T u_{0}\right), \quad \zeta \in \widetilde{P}\left[V\left(u_{0}\right)\right] .
$$

Set $S_{n} \zeta=R\left(\zeta-\lambda T_{n} \zeta+\lambda f-\lambda T u_{0}\right)$ and consider $S_{n}$ as a mapping from $\widetilde{P}\left[V\left(u_{0}\right)\right]$ into $\widetilde{P}\left[V\left(u_{0}\right)\right]$. We have, because of $(2.3)$

$$
\left\|S_{n} \zeta-S_{n} \varkappa\right\| \leqq\left\|\left(I-\lambda T_{n}\right)(\zeta-\varkappa)\right\| \leqq\left\|I-\lambda T_{n}\right\|\|\zeta-\varkappa\| .
$$

It is easily seen that, by assuming $\lambda<2 n^{-1}\left\|T_{n}\right\|^{-2}$, we have $\left\|I-\lambda T_{n}\right\|<1$. Thus $S_{n}$ is a contraction, and (2.5) has one and only one solution. Let us denote by $z_{n}$ the unique solution of (2.4). Suppose we have proved that $\left\{z_{n}\right\}$ contains a bounded subsequence. We may extract from $\left\{z_{n}\right\}$ a weakly convergent subsequence, which we still denote by $\left\{z_{n}\right\}$. Let $\widetilde{P} z$ be the weak limit of $\left\{z_{n}\right\}$. We may suppose $z \in V\left(u_{0}\right)$. Set $u=z+u_{0}$. Keeping in mind that $(T v, v)$ is lower semi- 
continuous with respect to weak convergence and setting $\widetilde{P}\left(v-u_{0}\right)=w(v \in V)$, we have

$$
\begin{aligned}
(T u, v-u) & =\left(T z,\left(v-u_{0}\right)-\left(u-u_{0}\right)\right)+\left(T u_{0}, v-u\right) \\
& =(T \widetilde{P} z, w-\widetilde{P} z)+\left(T u_{0}, v-u\right) \geqq-\operatorname{minlim}_{n \rightarrow \infty}\left(T z_{n}, z_{n}-w\right)+\left(T u_{0}, v-u\right) \\
& \geqq-\lim _{n \rightarrow \infty}\left\{\left(f-T u_{0}, z_{n}-w\right)-\frac{1}{n}\left(z_{n}, z_{n}-w\right)\right\}+\left(T u_{0}, v-u\right) \\
& =\left(f-T u_{0}, w-\widetilde{P} z\right)+\left(T u_{0}, v-u\right) \\
& =\left(f-T u_{0}, v-u_{0}-z\right)+\left(T u_{0}, v-u\right)=(f, v-u) .
\end{aligned}
$$

Thus we have to prove that $\left\{z_{n}\right\}$ contains a bounded subsequence. Set $\sigma_{n}=\left\|z_{n}\right\|$ and suppose that $\lim _{n \rightarrow \infty} \sigma_{n}=+\infty$.

From (2.4), assuming $w=\widetilde{P}\left(v-u_{0}\right)(v \in V)$, we deduce that

$$
c\left\|P z_{n}\right\|^{2} \leqq\left(z_{n}, T^{*} v-T^{*} u_{0}\right)+\frac{1}{n}\left(z_{n}, v-u_{0}\right)+\left(f-T u_{0}, z_{n}\right)-\left(f-T u_{0}, v-u_{0}\right) .
$$

Set $x_{n}=\sigma_{n}^{-1} z_{n}$. Then

$$
\begin{aligned}
c \sigma_{n}^{2}\left\|P x_{n}\right\|^{2} \leqq & \sigma_{n}\left(x_{n}, T^{*} v-T^{*} u_{0}\right)+\frac{\sigma_{n}}{n}\left(x_{n}, v-u_{0}\right) \\
& +\sigma_{n}\left(f-T u_{0}, x_{n}\right)-\left(f-T u_{0}, v-u_{0}\right) .
\end{aligned}
$$

Hence $\lim _{n \rightarrow \infty}\left\|P x_{n}\right\|=0$.

We have $\widetilde{P}=P+Q_{0}+Q_{1}$ and $P, Q_{0}, Q_{1}$ are mutually orthogonal projectors. Hence

$$
\left\|P x_{n}\right\|^{2}+\left\|Q_{0} x_{n}\right\|^{2}+\left\|Q_{1} x_{n}\right\|^{2}=1 .
$$

Thus we may extract from $\left\{x_{n}\right\}$ a subsequence, which we still denote by $\left\{x_{n}\right\}$, such that

Set $\varrho=\varrho_{0}+\varrho_{1}$. Since

$$
\begin{gathered}
\lim _{n \rightarrow \infty} Q_{0} x_{n}=\varrho_{0} \in K(T), \quad \lim _{n \rightarrow \infty} Q_{1} x_{n}=\varrho_{1} \in L_{1}, \\
\left\|\varrho_{0}\right\|^{2}+\left\|\varrho_{1}\right\|^{2}=1 .
\end{gathered}
$$

$$
t \varrho=\lim _{n \rightarrow \infty} t x_{n} \quad(t>0)
$$

and, for $n$ large enough, $t x_{n} \in \widetilde{P}\left[V\left(u_{0}\right)\right]$, then $t \varrho \in \widetilde{P}\left[V\left(u_{0}\right)\right]$. Let $\varrho^{\prime}$ be a vector of $V\left(u_{0}\right)$ such that $\widetilde{P} \varrho^{\prime}=\varrho$. We have $\varrho^{\prime}=\varrho+\left(\varrho^{\prime}-\varrho\right)$. Since $\varrho \in L_{1} \oplus K(T)$ and $\varrho^{\prime}-\varrho \in L$, we have $\varrho^{\prime} \in N\left(T+T^{*}\right) \cap V\left(u_{0}\right)$. If $\varrho_{0}=0$, then $\varrho^{\prime} \in N(T) \cap V\left(u_{0}\right)$. Eq. (2.7) implies $\varrho_{1} \neq 0$ and, in consequence, $F\left(\varrho_{1}\right)=F\left(\varrho_{1}\right)+F\left(\varrho^{\prime}-\varrho_{1}\right)=F\left(\varrho^{\prime}\right)<0$. Then

$$
\lim _{n \rightarrow \infty}\left(f+T^{*} v, Q_{0} x_{n}+Q_{1} x_{n}\right)<0 .
$$

If $\varrho_{0} \neq 0$, then $Q_{0} \varrho^{\prime} \neq 0$ and, assuming $v=v_{\varrho^{\prime}}$, we have $\left(f, \varrho^{\prime}\right)+\left(T^{*} v_{\varrho^{\prime}}, \varrho^{\prime}\right)<0$. Hence

$$
\begin{aligned}
(f, \varrho)+\left(T^{*} v_{\varrho^{\prime}}, \varrho\right)= & (f, \varrho)+\left(T^{*} v_{\varrho^{\prime}}, \varrho\right)+\left(f, \varrho^{\prime}-\varrho\right) \\
& +\left(v_{\varrho^{\prime}}, T\left(\varrho^{\prime}-\varrho\right)\right)=\left(f, \varrho^{\prime}\right)+\left(T^{*} v_{\varrho^{\prime}}, \varrho^{\prime}\right)<0,
\end{aligned}
$$

and (2.8) holds also in this case, provided we assume $v=v_{\varrho^{\prime}}$.

From (2.6), where $v$ is any fixed vector of $V$ if $\varrho_{0}=0$ and $v=v_{\varrho^{\prime}}$ if $\varrho_{0} \neq 0$, we deduce that

$$
\begin{aligned}
c \sigma_{n}\left\|P x_{n}\right\|^{2} \leqq & \left(P x_{n}, T^{*} v-T^{*} u_{0}\right)+\frac{1}{n}\left(x_{n}, v-u_{0}\right) \\
& +\left(P x_{n}, f-T u_{0}\right)+\left(Q_{0} x_{n}+Q_{1} x_{n} f+T^{*} v\right)-\frac{1}{\sigma_{n}}\left(f-T u_{0}, v-u_{0}\right),
\end{aligned}
$$


which is absurd since the minlim of the left hand side is nonnegative while the limit of the right hand side is negative.

Remark. If the set $N\left(T+T^{*}\right) \cap V\left(u_{0}\right)$ is bounded [and (ii) holds], the unilateral problem (2.1) has a solution for any given $F$. In particular this condition is satisfied if $V$ is bounded. In this case the condition (1.1) is not needed but only $B(v, v) \geqq 0$. In fact, if $V$ is bounded, the sequence $\left\{z_{n}\right\}$ is weakly compact.

The following theorem proves the necessity of condition i) of Theorem 2.I.

2.II If the unilateral problem (2.1) has a solution $u$, for any $u_{0} \in V$ and any $\varrho$ such that

$$
\varrho \in N(T) \cap V\left(u_{0}\right), \quad p\left\{Q_{1} \varrho, Q_{1}\left[N(T) \cap V\left(u_{0}\right)\right]\right\}=+\infty,
$$

one must have $F(\varrho)<0$.

The proof is exactly the same as for Theorem 1.III.

Concerning condition ii), the same example as given in the symmetric case proves that if condition ii) is dropped the theorem may fail to be true.

With respect to condition iii) we can only prove the following

2.III If for every $u_{0} \in V$ and for some $\varrho$ satisfying the conditions

$$
\begin{gathered}
Q_{0} \varrho \neq 0, \quad \varrho \in N\left(T+T^{*}\right) \cap V\left(u_{0}\right), \\
p\left\{\left(Q_{0}+Q_{1}\right) \varrho,\left(Q_{0}+Q_{1}\right)\left[N\left(T+T^{*}\right) \cap V\left(u_{0}\right)\right]\right\}=+\infty,
\end{gathered}
$$

we have

$$
F(\varrho)+B(\varrho, v)>0
$$

for every $v \in V$, then the unilateral problem (2.1) has no solution.

Suppose, contrarywise, that problem (2.1) has a solution $u$. Let us have, for every $t>0,\left(Q_{0}+Q_{1}\right) \varrho(t)=t\left(Q_{0}+Q_{1}\right) \varrho, \varrho(t) \in N\left(T+T^{*}\right) \cap V\left(u_{0}\right)$. Then

$$
\left(T u+T^{*} u, \varrho(t)+u_{0}-u\right) \geqq t\left(f+T^{*} u, \varrho(t)\right)+\left(f+T^{*} u, u_{0}-u\right) .
$$

Since $T \varrho(t)+T^{*} \varrho(t)=0,\left(f+T^{*} u, \varrho(t)\right)=t\left(f+T^{*} u, \varrho\right)$, we conclude that

which implies

$$
\left(T u, u_{0}-u\right)-\left(f, u_{0}-u\right) \geqq t\left(f+T^{*} u, \varrho\right)
$$

$$
\left(f+T^{*} u, \varrho\right)=F(\varrho)+B(\varrho, u) \leqq 0
$$

in contradiction with (2.10).

2.IV If $u$ is a solution of the unilateral problem (2.1) the vector $P u$ is uniquely determined by $F$.

Arguing as in the proof of Theorem 1.V, we get (1.5) and the proof of Theorem 2.IV.

2.V If $B(v, v)$ is coercive on $H$, i.e.

$$
c\|v\|^{2} \leqq B(v, v) \quad \forall v \in H,
$$

the unilateral problem (2.1) has only one solution for any given $F$. If we denote by $G(F)$ the solution of the unilateral problem (2.1), $G$ is a Lipschitz-continuous mapping from $H^{*}$ (dual space of $H$ ) into $V$.

Under the assumed hypotheses, problem (2.1) has a solution for every given $F \in H^{*}$. In this case, since $P \equiv I$, the inequality (1.4) gives

$$
c\left\|u-u^{\prime}\right\| \leqq\left\|F-F^{\prime}\right\|,
$$


which proves uniqueness and, moreover,

$$
\left\|G(F)-G\left(F^{\prime}\right)\right\| \leqq c^{-1}\left\|F-F^{\prime}\right\| .
$$

Remark. For the uniqueness of the solution of the unilateral problem (2.1), it is sufficient the quadratic form $B(v, v)$ be strictly positive on $H$. In fact, if $u$ and $u^{\prime}$ are two solutions of $(2.1)$, we have $B\left(u, u^{\prime}-u\right) \geqq F\left(u^{\prime}-u\right), B\left(u^{\prime}, u-u^{\prime}\right) \geqq$ $F\left(u-u^{\prime}\right)$. Hence $B\left(u-u^{\prime}, u-u^{\prime}\right) \leqq 0$, which implies $u=u^{\prime}$.

3. Unilateral problems for elliptic operators. Let $A$ be a bounded domain of the cartesian space $X^{r}$, and let $B(u, v)$ be the bilinear form considered in Sect. 5 of E.T.E.

$$
B(u, v)=(-1)^{m} \sum_{|p|,|q|}^{c, m}(-1)^{|p|} \int_{A} a_{p q}(x) D^{q} u D^{p} v d x .
$$

We now assume that the $n \times n$ matrices $a_{p q}$ have real entries which are bounded and measurable functions in $A$; $u$ and $v$ are real $n$-vector valued functions of $H_{m}(A)$ which we now consider as a real Hilbert space. ${ }^{4}$

Let $H$ be a closed, linear subspace of $H_{m}(A)$, and suppose that the bilinear form, when restricted to $H \times H$, satisfies Hypotheses I and II considered in Sects. 1 and 2 .

(I) Denote by $P$ the projector of $H$ onto the orthogonal complement of the kernel of $B(v, v)$ (restricted to $H)$. A positive constant $c$ exists such that

$$
B(v, v) \geqq c\|P v\|_{m}, \quad \forall v \in H .
$$

(II) The kernel of $B(v, v)$ is finite dimensional.

Let $V$ be a closed convex set of $H$. We can apply the theory of Sects. 1 and 2 to this particular case and give existence theorems for the unilateral problem

$$
(-1)^{m} \sum_{|p|,|q|}^{0, m}(-1)^{|p|} \int_{A} a_{p q}(x) D^{q} u D^{p}(v-u) d x \geqq F(v-u), \quad u \in V, \forall v \in V,
$$

where $F$ is a given bounded linear functional defined on $H$.

Suppose that the following further hypotheses are satisfied.

(III) The convex set $V$ contains $\stackrel{\circ}{H}_{m}(A)$.

(IV) The differential operator

$$
L(x, D)=(-1)^{m} D^{p} a_{p q} D^{q}, \quad(0 \leqq|p| \leqq m), \quad(0 \leqq|q| \leqq m)
$$

is elliptic for every $x \in A$.

In addition assume that $a_{p q}(x) \in C^{\infty}(A)$ and $F(v)=(f, v)_{0}{ }^{5}$ for any $v \in \stackrel{H}{m}_{m}(A)$, where $f$ is a function of $H_{v}(A)$. We have the following theorems:

3.I Under the assumed hypotheses, if $u$ is a solution of the unilateral problem (3.1), then $u$ belongs to $H_{2 m+v}(B)$, where $B$ is any domain such that $B \subset A$, and $u$ is a solution in $A$ of the differential system $L u=f$.

If $v \in \AA^{\infty}(A)$ and $t$ is an arbitrary real constant, from (3.1) we deduce $t B(u, v)$ $B(u, u) \geqq t(f, v)_{0}-(f, u)_{0}$. Hence, since $t$ is arbitrary, $B(u, v)=(f, v)_{0}$, and after integrations by parts we obtain

$$
\int_{A} u L^{*} v d x=\int_{A} f v d x .
$$

4 We use in this paper concepts and notations already introduced in E.T.E.

$5(\bullet, \bullet)_{0}$ denotes scalar product in $H_{0}(A) \equiv \mathscr{L}^{2}(A)$. 
From Theorem 3.I of E.T.E. we get the proof.

\section{II The space $N(T)$ consists in all the functions @ such that}

$$
L \varrho=0, \quad \varrho \in \mathscr{U}_{H} \cdot{ }^{6}
$$

We obtain the proof of this theorem by observing that $\varrho \in N(T)$ if and only if

$$
B(\varrho, v)=0, \quad \varrho \in H, \forall v \in H .
$$

From the results of Sect. 6 of E.T.E. it follows that $\varrho \in N(T)$ when and only when $\varrho$ satisfies (3.2).

We have a similar result for characterizing the functions $\varrho$ which belong to $N\left(T+T^{*}\right)$. In fact we have to use the same argument but referred to the symmetric bilinear form $B(u, v)+B(v, u)$. As a consequence we deduce that $N\left(T+T^{*}\right)$ is formed by all the solutions of the equation $L u+L^{*} u=0$ that belong to a certain subspace $\mathscr{U}_{H}^{\prime}$ of $H ; L^{*}$ is the operator $L^{*}=(-1)^{m} D^{p} \alpha_{p q} D^{q}$ where $\alpha_{p q}=(-1)^{|p|+|q|} \bar{a}_{q p}$.

Let $A^{\prime}$ be an open subset of $A$ and consider the function space $\stackrel{\circ}{H}_{m}\left(A^{\prime}\right)$. We may consider $\stackrel{\circ}{H}_{m}\left(A^{\prime}\right)$ as a subspace of $\stackrel{\circ}{H}_{m}(A)$ if we suppose that each function $u$ of $\stackrel{\circ}{H}_{m}\left(A^{\prime}\right)$ is continued into the whole set $A$ by assuming $u=0$ in $A-A^{\prime}$.

Suppose we have, instead of (III) the weaker hypothesis

(III') The convex set $V$ contains $\stackrel{\leftrightarrow}{H}_{m}\left(A^{\prime}\right)$.

If we retain unchanged the other hypotheses on the ellipticity of $L$ and on the smoothness of the coefficients $a_{p q}$ and of $f$ (or, at most, we restrict them to $A^{\prime}$ ), Theorem 3.I still holds, provided we read $A^{\prime}$ instead of $A$ in its statement.

Let us now suppose that the domain $A$ is $C^{\infty}$-smooth at the point $x^{0}$ of $\partial A$ (see E.T.E., Sect. 6). Let $I_{0}$ be a neighborhood of $x^{0}$ containing the neighborhood $I$ of $x^{0}$ which is involved in the definition of $C^{\infty}$-smoothness. Let $V_{0}$ be a class of functions $v$ which are defined on the whole space $X^{r}$ and which have their supports in $I_{0}$. If $B$ is any set of $X^{r}$, we shall denote by $V_{0}(B)$ the class of functions on $B$ obtained by taking the restriction to $B$ of every $v \in V_{0}$. Let us suppose that: (i) $V_{0}(A)$ is a closed linear subspace of $H_{m}(A)$; (ii) $V_{0}\left(A \cap I_{0}\right) \supset \stackrel{H}{H}_{m}\left(A \cap I_{0}\right)$; (iii) for every $v \in V_{0}\left(A \cap I_{0}\right)$, the following inequality holds

$$
B(v, v) \geqq \gamma_{0}\|v\|_{m, A}^{2} \quad\left(\gamma_{0}>0\right) ;
$$

(iv) Hypothesis $(\mathrm{IV})_{x_{0}}$ of Sect. 6 of E.T.E. is satisfied for the class $V_{0}(A)$.

Let us now assume that

(III") The convex set $V$ contains the subspace $V_{0}(A)$.

Let us remark that Hypothesis $\left(\mathrm{III}^{\prime \prime}\right)$ implies Hypothesis $\left(\mathrm{III}^{\prime}\right)$ (with $A^{\prime}=A \cap I_{0}$ ), because of ii), and implies Hypothesis (IV) (restricted to $A \cap I_{0}$ ), because of inequality (3.3) (see E.T.E. Theorem 5.II).

Let us assume that $a_{p q} \in C^{\infty}\left(X^{r}\right)$ and that for every $v \in V_{0}(A)$ we have $F(v)=$ $(f, v)_{0}$ where $f$ is a function of $H_{v}(A)$. The following theorem holds.

3.III Under the assumed hypotheses, every solution $u$ of the unilateral problem (3.1) belongs to $H_{2 m+v}(B)$ for any domain $B$ such that $\bar{B} C \bar{A} \cap(A \cup I)$.

Arguing as in the proof of Theorem 3.I we see that $B(u, v)=(f, v)_{0}$ for every $v \in V_{0}(A)$. Then the proof of our theorem follows from Theorem 6.VI of E.T.E.

\footnotetext{
6 For the definition of $\mathscr{U}_{H}$ see E.T.E., Sect. 6 (definition of problem (P)).
} 
Theorem 3.I and the analogous theorem relative to Hypothesis (III'), give results concerning the interior regularity of $u$, while Theorem 3.III concerns the boundary regularization of $u$. Of course these theorems are obtained under the strong assumption that $V$ contains linear subspaces such as to permit us to carry over the results of the regularization theory developed for linear problems. When this assumption is not satisfied, the regularization theory becomes much more difficult, as we shall see later, and the solution has, in general, only a mild degree of regularity even if the data are very smooth.

Remark. Suppose that a solution $u$ of (3.1) exists such that $B(u, u)=F(u)$. Then Theorem 3.I [3.III] still holds for such a solution if we substitute for Hypothesis (III) [III'] the weaker one: the convex set $V$ contains the closed ball $\|w\| \leqq \varepsilon(\varepsilon>0)$ of $\stackrel{\circ}{H}_{m}(A)\left[V_{0}(A)\right]$.

The proof is readily obtained by an easy modification of the proof of Theorem 3.I.

4. General definition for the convex set $\boldsymbol{V}$. Let us now consider three definitions for the convex set $V$ that include the unilateral problems which generally arise in the theory of elasticity.

Let $\Phi_{h}\left(x, z_{0}, z_{1}, \ldots, z_{s}\right)(h=1, \ldots, l)$ be a real valued function defined for $x \in X^{r}$ and for every choice of the vectors $z_{0}, \ldots, z_{s}$. We suppose that $z_{0}, \ldots, z_{s}$ are such that for every $n$-vector valued function $v \in C^{\infty}\left(X^{r}\right)$ we may consider the functions

for $0 \leqq|p| \leqq m$.

$$
\Phi_{h}\left[x, v(x), \ldots, D^{p} v(x), \ldots\right]
$$

We suppose that every $\Phi_{h}$ depends continuously on the variables $x, z_{0}, \ldots, z_{s}$. Moreover we suppose that, for $t_{1} \geqq 0, t_{2} \geqq 0$ and $t_{1}+t_{2}=1$ and for any choice of $x, z_{\mathbf{0}}^{(1)}, \ldots, z_{s}^{(1)}, z_{\mathbf{0}}^{(2)}, \ldots, z_{s}^{(2)}$,

$$
\begin{gathered}
\Phi_{h}\left(x, t_{1} z_{0}^{(1)}+t_{2} z_{0}^{(2)}, \ldots, t_{1} z_{s}^{(1)}+t_{2} z_{s}^{(2)}\right) \leqq t_{1} \Phi_{h}\left(x, z_{0}^{(1)}, \ldots, z_{s}^{(1)}\right) \\
+t_{2} \Phi_{h}\left(x, z_{0}^{(2)}, \ldots, z_{s}^{(2)}\right) \quad \Phi_{h}(x, 0, \ldots, 0) \leqq 0
\end{gathered}
$$

Let $H$ be a closed linear subspace of $H_{m}(A)$, and let $A_{h}$ be a measurable subset of $A(h=1, \ldots, l)$.

Let $\varphi_{h}(x)(h=1, \ldots, l)$ be a real valued function, nonnegative and measurable in $A_{h}$.

We define $V$ as follows.

a) $V$ is the set of all the functions $v$ of $H$ such that almost everywhere on $A_{h}$

$$
\Phi_{h}\left[x, v(x), \ldots, D^{p} v(x), \ldots\right] \leqq \varphi_{h}(x) \quad(h=1, \ldots, l) .
$$

From (4.1) we deduce that $V$ is not empty and is convex. Let $\left\{v_{n}\right\}$ be a converging sequence (in the topology of $H_{m}(A)$ ) of functions belonging to $V$. Let $v$ be the limit of this sequence. Since from $\left\{v_{n}\right\}$ we can extract a subsequence, which we still denote by $\left\{v_{n}\right\}$, such that $\left\{D^{p} v_{n}\right\}$ converges to $D^{p} v$ almost everywhere on $A$, we see that the function $v$ satisfies condition (4.2), i.e. belongs to $V$. Thus we have proved that $V$ is closed.

Thus we may apply to the bilinear form $B(u, v)$ and to the convex set $V$ the theory developed in the preceding sections. In particular if we choose $l=1$,

$$
\Phi_{1}\left(x, v, \ldots, D^{p} v, \ldots\right)=\sum_{|p|}^{0, m}\left|D^{p} v\right|^{2},
$$


take $A_{1} \equiv A$ and suppose that $\varphi_{1}(x)$ is bounded in $A$, we have a bounded closed convex set $V$. In this case the unilateral problem (3.1) has a solution for any given $F$.

Let us now consider a second general definition for the convex set $V$. Let us suppose that $A$ is properly regular (see E.T.E. Sect. 2) and let $\Psi_{h}(x, z)\left(h=1, \ldots, l^{\prime}\right)$ be a real valued function defined for $x \in \partial A$ and for any choice of the vector $z$. We suppose that $z$ has as many components as needed for considering the function

$$
\Psi_{h}[x, \tau v(x)] \quad\left(h=1, \ldots, l^{\prime}\right)
$$

where $v(x)$ is an $n$-vector valued function belonging to $C^{m}(\bar{A})$ and $\tau$ is the "boundary value operator" defined in Sect. 2 of E.T.E. We suppose that the boundary $\partial A$ is decomposable into a finite number of non-overlapping differentiable $(r-1)$ cells ${ }^{7}$ and that $\Psi_{h}(x, z)$ is continuous when $x$ varies in any of these cells and $z$ varies arbitrarily. Moreover for $t_{1} \geqq 0, t_{2} \geqq 0, t_{1}+t_{2}=1$ and any $x, z^{(1)}, z^{(2)}$, we have

$$
\Psi_{h}\left(x, t_{1} z^{(1)}+t_{2} z^{(2)}\right) \leqq t_{1} \Psi_{h}\left(x, z^{(1)}\right)+t_{2} \Psi_{h}\left(x, z^{(2)}\right) \quad \Psi_{h}(x, 0) \leqq 0 .
$$

Let $H$ be a closed linear subspace of $H_{m}(A)$ and $\Sigma_{h}\left(h=1, \ldots, l^{\prime}\right)$ a subset of $\partial A$ composed by non-overlapping differentiable $(r-1)$-cells. Let $\psi_{h}(x)$ be a real valued function nonnegative and measurable on $\Sigma_{h}$.

We now define $V$ as follows. have

$\beta$ ) $V$ is the set of all the functions $v$ of $H$ such that almost everywhere on $\Sigma_{h}$ we

$$
\Psi[x, \tau v(x)] \leqq \psi_{h}(x) \quad\left(h=1, \ldots, l^{\prime}\right) .^{8}
$$

As for the definition $\alpha$ ) it is easy to prove that $V$ is non-empty, closed and convex.

As third definition we take the one corresponding to the set which is the intersection of the two convex sets defined, respectively, by $\alpha$ ) and by $\beta$ ).

$\gamma) V$ is the set of all the functions of $H$ satisfying conditions (4.2) and (4.3).

Since the intersection of two closed convex sets is closed and convex, so is the set $V$ defined by $\gamma$ ).

5. Unilateral problems for an elastic body. Let $A$ be a bounded domain of $X^{r}$ which we suppose properly regular and with a boundary $\partial A$ that can be decomposed into a finite number of non-overlapping $(r-1)$-cells of class $C^{n}(n \geqq 1)$. Let $A$ represent the natural configuration of an $r$-dimensional elastic body, which we denote by the same letter $A$. Using the notations of Sect. 12 of E.T.E., for every pair of $r$-vectors $u, v$ of $H_{1}(A)$ we consider the bilinear form

$$
B(u, v)=\int_{A} a_{i h, j k}(x) u_{i / h} u_{j / k} d x,
$$

7 It is convenient to recall here the definition of a differentiable $(r-1)$-cell. Let $x=x(t) \equiv$ $\left[x_{1}(t), \ldots, x_{r}(t)\right]$ be a $r$-vector valued function defined in the closed domain $T^{r-1}, 0 \leqq t_{i} \leqq 1$ $(i=1, \ldots, r-1) 0 \leqq t_{1}+\cdots+t_{r-1} \leqq 1$ of the $(r-1)$-dimensional space, satisfying the following conditions: i) $x=x(t)$ belongs to the class $C^{n}\left(T^{r-1}\right)(n \geqq 1)$; ii) the jacobian matrix $\partial x / \partial t$ has rank $r-1$ at every point of $T^{r-1}$; iii) the function $x=x(t)$ is univalent in $T^{r-1}$. The range $\Gamma$ of the function $x=x(t)$ is, by definition, a differentiable $(r-1)$-cell of class $C^{n}$. The set of all the points of $\Gamma$, which correspond to the boundary $\partial T^{r-1}$ of $T^{r-1}$, is the border $\partial \Gamma$ of $\Gamma$. When we say that two $(r-1)$-cells are non-overlapping, we mean that they have in common, at most, points of their borders.

8 It is evident that now the concepts "measurable function" and "almost everywhere" must be understood with respect to the hypersurface measure on $\partial A$. 
where the functions $a_{i h, j k}(x)$ are the ones introduced in Sect. 12 of E.T.E. and satisfy the hypotheses stated in that section. Let $f$ be a given $r$-vector belonging to $H_{0}(A)$ and $g$ a given $r$-vector belonging to $\mathscr{L}^{2}(\partial A)$. Let the energy functional to be associated with the body $A$ be the following

where

$$
\mathscr{I}(v)=\frac{1}{2} B(v, v)-F(v),
$$

$$
F(v)=\int_{A} f_{i} v_{i} d x+\int_{\partial A} g_{i} v_{i} d \sigma .
$$

Let $\Phi_{h}\left(x ; z_{0} ; z_{1}\right)(h=1, \ldots, l)$ be a real valued function depending on the $r$-vector $x$, the $r$-vector $z_{0}$, and the $r^{2}$-vector $z_{1}$, defined in the whole $\left(2 r+r^{2}\right)$ dimensional cartesian space and continuous at every point $\left(x ; z_{0} ; z_{1}\right)$.

For the functions $\Phi_{h}$ we assume that condition (4.1) is satisfied, i.e. for $t_{1} \geqq 0, t_{2} \geqq 0, t_{1}+t_{2}=1$ and any choice of $x, z_{0}^{(1)}, z_{1}^{(1)}, z_{0}^{(2)}, z_{1}^{(2)}$, we have

$$
\begin{aligned}
\Phi_{h}\left(x ; t_{1} z_{\mathbf{0}}^{(\mathbf{1})}+t_{\mathbf{2}} z_{\mathbf{0}}^{(2)} ; t_{\mathbf{1}} z_{\mathbf{1}}^{(\mathbf{1})}+t_{\mathbf{2}} z_{1}^{(\mathbf{2})}\right) & \leqq t_{\mathbf{1}} \Phi_{h}\left(x ; z_{0}^{(\mathbf{1})} ; z_{1}^{(\mathbf{1})}\right)+t_{\mathbf{2}} \Phi_{h}\left(x ; z_{\mathbf{0}}^{(\mathbf{2})} ; z_{\mathbf{1}}^{(\mathbf{2})}\right) \\
\Phi_{h}(x ; 0 ; 0) & \leqq 0 .
\end{aligned}
$$

Let $\varphi_{h}(x)$ be a nonnegative, bounded measurable function in the subdomain $A_{h}$ of $A$.

We suppose that the elastic body is subjected to the following internal constraints

$$
\Phi_{h}\left(x ; v ; v_{1}, \ldots, v_{\eta}\right) \leqq \varphi_{h}(x)
$$

almost everywhere in $A_{h}(h=1, \ldots, l)$.

Let $\Psi_{h}(x, z)\left(h=1, \ldots, l^{\prime}\right)$ be a real valued function defined for $x \in \partial A$ and for every $r$-vector $z$. Let the function $\Psi_{h}(x, z)$ be continuous when $x$ varies in each $(r-1)$-cell of $\partial A$ and $z$ in the $r$-dimensional cartesian space. Moreover for $t_{1} \geqq 0, t_{2} \geqq 0, t_{1}+t_{2}=1$, for each $x \in \partial A$ and every choice of $z^{(\mathbf{1})}$ and $z^{(\mathbf{2})}$

$$
\Psi_{h}\left(x, t_{1} z^{(\mathbf{1})}+t_{2} z^{(2)}\right) \leqq t_{1} \Psi_{h}\left(x, z^{(1)}\right)+t_{2} \Psi_{h}\left(x, z^{(2)}\right) \quad \Psi_{h}(x, 0) \leqq 0 .
$$

Let $\Sigma_{h}\left(h=1, \ldots, l^{\prime}\right)$ be a subset of $\partial A$ formed by non-overlapping differentiable $(r-1)$-cells of $\partial A$. Let $\psi_{h}(x)$ be a nonnegative bounded measurable function in $\Sigma_{h}$.

The elastic body be subjected to the following boundary constraints

$$
\Psi_{h}(x, \tau v) \leqq \psi_{h}(x)
$$

almost everywhere on $\Sigma_{h}\left(h=1, \ldots, l^{\prime}\right)$.

Let $H$ be a closed linear subspace of $H_{1}(A)$.

The following one is a very general problem in the classical theory of elasticity.

To minimize the functional $\mathscr{I}(v)$ in the subset $V$ of $H$ formed by all the functions v that satisfy the conditions (5.1) and (5.2).

The problem consists in finding the equilibrium configuration of an elastic body subjected to the body forces determined by $f$, to the surface forces determined by $g$, and subjected to the bilateral constraints, imposed upon the admissible displacements by requiring that they belong to $H$, and to the unilateral constraints represented by (5.1) and (5.2).

It must be remarked that either (5.1) or (5.2), which are stated here as unilateral constraints, may turn out, in some particular case, to be bilateral, as we shall see by an interesting illustration. 
The problem stated, although very general in elasticity, is a particular case of the theory developed in the previous sections of this article. The convex set $V$ is the one determined by condition $\gamma$ ) of the preceding section. Of course, since we do not exclude either the case when $l^{\prime}=0$ or the case when $l=0$, we may have convex sets either of the kind $\alpha$ ) or of the kind $\beta$ ). If we take $l=l^{\prime}=0$, we have the problems discussed in Sect. 12 of E.T.E.

Of particular relevance is the case when $l=0, l^{\prime}=1 ; \Psi_{1}(x, z)=-v(x) z[v(x)$ is the unit inward normal to $\partial A$ ]; $\Sigma_{1} \equiv \Sigma$ [subset of $\partial A$ composed by non-overlapping $(r-1)$-cells] ; $\psi_{1}(x) \equiv 0 ; g \equiv 0$ on $\Sigma ; H=H_{1}(A)$. The unilateral constraint is now the following

$$
v_{i} \nu_{i} \geqq 0 \quad \text { a.e. on } \Sigma \text {. }
$$

This particular case defines what is nowadays known in the literature as the Signorini problem. It corresponds to the equilibrium problem of an elastic body, which in its natural configuration is supported by a rigid frictionless surface $\Sigma$. We shall study this problem in full detail in the sequel.

Let us now assume $l=2, l^{\prime}=0$. Let $\Sigma$ be a subset of $\partial A$ formed by non-overlapping $(r-1)$-cells. Set $g \equiv 0$ on $\Sigma$. Let $H$ be the subspace of $H_{1}(A)$ formed by the functions of $H_{1}(A)$ vanishing on $\partial A$. Assume

$$
\Phi_{1}\left(x ; v ; v_{/ 1}, \ldots, v_{/ r}\right) \equiv-\Phi_{2}\left(x ; v ; v_{/ 1}, \ldots, v_{/ r}\right) \equiv v_{i / i},
$$

$A_{1}=A_{2}=A$ and $\varphi_{1}(x) \equiv \varphi_{2}(x) \equiv 0$. In this case the two unilateral constraints given by (5.1) are equivalent to the unique bilateral constraint $v_{i / i}=0$ in $A$. This is the incompressibility condition for the elastic body $A$. The equilibrium problem concerns now an incompressible elastic body, fixed along the part $\Sigma$ of its boundary, subjected to given surface forces $\varphi$ on the remaining part of the boundary and to given body forces $f$ on $A$. Theorem 1.III gives readily an existence theorem for the minimum of $\mathscr{I}(v)$ in this particular case.

\section{Other examples of unilateral problems.}

(I) The Signorini problem for a scalar 2nd order elliptic operator. Let $A$ be the domain considered in Sect. 10 of E.T.E. and let $B(u, v)$ be the bilinear form defined in the example III of that section. Under the hypotheses there assumed we have $-B(v, v) \geqq c_{0}\|v\|_{1}^{2}$. Let $H=H_{1}(A)$ and $V$ the convex set defined by the condition $v \geqq 0$ on $\partial A$. For any given $f \in H_{0}(A)$ there exists one and only one solution of the unilateral problem

$$
\begin{gathered}
\int_{A}\left\{\alpha_{i j} \frac{\partial(v-u)}{\partial x_{i}} \frac{\partial u}{\partial x_{j}}-\beta_{i}(v-u) \frac{\partial u}{\partial x_{i}}-c(v-u) u\right\} d x \\
\geqq \int_{A} f(v-u) d x \quad u \in V, \forall v \in V .
\end{gathered}
$$

In this case the convex set $V$ satisfies the Hypothesis (III) of Sect. 3. Then $u \in H_{2}(B)$ for any domain $B$ such that $\bar{B} \subset A$ and satisfies in $A$ the differential equation

$$
\frac{\partial}{\partial x_{i}}\left[\alpha_{i j} \frac{\partial u}{\partial x_{j}}\right]+\beta_{i} \frac{\partial u}{\partial x_{i}}+c u+f=0
$$

Let us now suppose that $u \in H_{2}(A) \cdot{ }^{9}$ From (6.1) we easily deduce $-B(u, u)=$ $(f, u)_{0},-B(u, v) \geqq(f, v)_{0}$. Moreover we have (see E.T.E., Sect. 10, III)

$$
B(u, v)=-\int_{A} f v d x+\int_{\partial \dot{A}} v \varrho \frac{\partial u}{\partial \lambda} d \sigma .
$$

\footnotetext{
9 This will turn out to be true from the analysis we shall develop in Sects. 8 and 9.
} 
Then

$$
\int_{\partial A} v \varrho \frac{\partial u}{\partial \lambda} d \sigma \leqq 0, \quad \int_{\partial A} u \varrho \frac{\partial u}{\partial \lambda} d \sigma=0 .
$$

By the arbitrariness of $v$ we deduce that $u$ satisfies almost everywhere on $\partial A$ the conditions:

$$
u \geqq 0, \quad \frac{\partial u}{\partial \lambda} \leqq 0, \quad u \frac{\partial u}{\partial \lambda}=0,
$$

which - provided they hold in the whole of $\partial A$-lead to the ambiguous boundary conditions

$$
\text { either }\left\{\begin{array} { c } 
{ u > 0 } \\
{ \frac { \partial u } { \partial \lambda } = 0 }
\end{array} \quad \text { or } \left\{\begin{array}{c}
u=0 \\
\frac{\partial u}{\partial \lambda} \leqq 0.10
\end{array}\right.\right.
$$

(II) Membrane fixed along its boundary and stretched over an obstacle. Let $A$ be a bounded domain of $X^{r}$. Assume

$$
B(u, v)=\int_{A} u_{/ i} v_{/ i} d x
$$

$(u, v$ real valued functions).

Let $\varphi(x)$ be a continuous function defined in $\bar{A}$ which takes nonpositive values on $\partial A$. Assume $H=\stackrel{\circ}{H}_{1}(A)$, and let the closed convex set $V$ be defined by the condition $u \geqq \varphi$ in $A .^{11}$

There exists one and only one solution $u$ of the unilateral problem

$$
\int_{A} u_{i i}\left(v_{/ i}-u_{/ i}\right) d x \geqq 0 \text {. }
$$

The function $u$ minimizes in $V$ the Dirichlet integral

$$
\int_{A} v_{/ i} v_{/ i} d x
$$

in the set $V$. In the case when $r=2, u$ gives the equilibrium configuration of a membrane (coinciding with the domain $A$ in its natural configuration) which is stretched over an obstacle represented by the function $\varphi(x)$.

(III) Elastic plastic torsion problem. This problem leads to a unilateral problem relative to a bounded domain $A$ of $X^{r}$. Also in this case, the bilinear form $B(u, v)$ is given by (6.2). $H$ is again $\stackrel{\circ}{H}_{1}(A)$. The closed convex set $V$ is defined by the condition

$$
\left|u_{/ i} u_{/ i}\right| \leqq a
$$

where $a$ is a positive constant. From the general theory we know that there exists one and only one solution of the unilateral problem.

$$
\int_{A} u_{/ i}\left(v_{/ i}-u_{/ i}\right) d x \geqq b \int_{A}(v-u) d x
$$

10 Boundary conditions of this kind were indicated for the first time by SIGNORINI in his problem in elasticity. He proposed the term "ambiguous boundary conditions" because it is not known a priori what set of conditions at a given point of the boundary is satisfied by the solution $u$ of the problem.

11 For a more general formulation of this problem see [26]. 
( $b$ given constant). The function $u$ minimizes in $V$ the functional

$$
\frac{1}{2} \int_{A} v_{/ i} v_{1 i} d x-b \int_{A} v d x .
$$

(IV) Clamped plate, partially supported on a subdomain. Let $A$ be a bounded domain of the plane $x, y$, and let $B(u, v)$ be the bilinear form defined in Sect. 11 of E.T.E. which is used for proving Theorem 11.I. Let $H \equiv \stackrel{H}{2}_{2}(A)$. Let $A_{0}$ be a subdomain of $A$, and let $V$ be the closed convex set defined by the condition $v \geqq 0$ in $A_{\mathbf{0}}$. There exists one and only one solution $u$ of the unilateral problem

$$
\int_{\boldsymbol{A}}\left(\frac{\partial^{2} u}{\partial x^{2}} \frac{\partial^{2}(v-u)}{\partial x^{2}}+2 \frac{\partial^{2} u}{\partial x \partial y} \frac{\partial^{2}(v-u)}{\partial x \partial y}+\frac{\partial^{2} u}{\partial y^{2}} \frac{\partial^{2}(v-u)}{\partial y^{2}}\right) d x d y \geqq \int_{A} f(v-u) d x d y
$$

( $f$ is a function of $H_{0}(A)$ ).

The function $u$ minimizes in $V$ the functional

$$
\frac{1}{2} \int_{A}\left\{\left(\frac{\partial^{2} v}{\partial x^{2}}\right)^{2}+2\left(\frac{\partial^{2} v}{\partial x \partial y}\right)^{2}+\left(\frac{\partial^{2} v}{\partial y^{2}}\right)^{2}\right\} d x d y-\int_{A} f v d x d y .
$$

Let us observe that in this example, if $A_{0}$ is a proper subdomain of $A$, the convex set $V$ satisfies the condition (III') of Sect. 3 for every domain $A^{\prime} C A-\bar{A}_{0}$ (if there is any). Then $u \in H_{4+v}\left(A^{\prime}\right)$ if $f \in H_{v}\left(A^{\prime}\right)$ and $\Delta_{4} v=f$ in $A^{\prime}$. Moreover if $\partial A$ is $C^{\infty}$-smooth in $x^{0}$, Hypothesis (III') of Sect. 3 is satisfied by assuming as $V_{0}$ the space $\stackrel{H}{2}_{2}\left(A \cap I_{0}\right)^{12}$ provided we can take $I_{0}$ at a positive distance from $A_{0}$. It follows that $u$ is smooth in the neighborhood of $x^{0}$ according to the smoothness of $f$.

(V) Elastic, perfectly locking body. $A$ is the domain considered in Sect. 5 and $B(u, v)$ the bilinear form defined in that section. $H$ is one of the following three spaces: $\stackrel{H}{1}_{1}(A), H_{1}(A), H_{\Sigma} \equiv\left\{v ; v \in H_{1}(A), v=0\right.$ on $\left.\Sigma\right\}$ ( $\Sigma$ is a subset of $\partial A$ formed by $(r-1)$-cells of $\partial A$ ).

Let $\varphi(\varepsilon)$ be a function depending on the symmetric tensor $\varepsilon \equiv\left\{\varepsilon_{i j}\right\}$ which is continuous for every $\varepsilon$, is a convex function of $\varepsilon$ and is such that $\varphi(0)<0$. that

Let $V$ be the closed convex subset of $H$ formed by all the functions $v$ such

$$
\varphi\left[\ldots, 2^{-1}\left(v_{j / i}+v_{i / j}\right), \ldots\right] \leqq 0 \quad \text { a.e. in } A .
$$

The kernel $N(T)$ of the quadratic form $B(v, v)$ is constituted by all the rigid displacements $\varrho=a+C x$ ( $a$ constant $r$-vector, $C$ skew-symmetric constant $r \times r$ matrix) belonging to $H$. From the Korn 2nd inequality, which we suppose to hold in $A$, it follows that Hypothesis (I of Sect. 1 is satisfied.13

If $R$ is the space of all the rigid displacements we have $N(T)=R \cap H$. Define $F(v)$ as in Sect. 5, taking $g \equiv 0$ on $\partial A$ if $H=\stackrel{\circ}{H}_{1}(A)$, and $g \equiv 0$ on $\partial A-\Sigma$ if $H=H_{\Sigma}$. A necessary condition for the existence of the minimum of $\mathscr{I}(v)$ in $V$ is $F(\varrho)=0$ for every $\varrho \in R \cap H$.

If $L, \widetilde{Q}$ and $\widetilde{P}$ are defined as in Sect. 1, and if we assume $u_{0}=0$, we see that $L=R \cap H$ and $\widetilde{P}[V] \subset V$. Hence, from Theorem 1.II, it follows that $\mathscr{I}(v)$ has a minimum in $V$. The minimizing function is determined up to a vector of $R \cap H$.

It must be remarked that $R \cap H \neq\{0\}$ only in the case when $H=H_{1}(A)$.

12 Of course we suppose, as usual, that each function of $\stackrel{\circ}{2}_{2}\left(A \cap I_{0}\right)$ is continued in the whole space and coincides with 0 outside of $A \cap I_{0}$.

13 We shall develop that in more detail in the next section. 
7. Existence theorem for the generalized Signorini problem. Let us now consider the problem stated in Sect. 5 under more specific assumptions on the functions $\Phi_{h}$ and $\Psi_{h}, \varphi_{h}, \psi_{h}$. We shall suppose that

(i) $\Phi_{h}\left(x ; z_{0} ; z_{1}\right)$ is a continuous function of $\left(x ; z_{0} ; z_{1}\right)$ for every choice of these variables;

(ii) $\Phi_{h}\left(x ; z_{0} ; z_{1}\right)$ for any $x \in X^{r}$ is a convex function of $\left(z_{0} ; z_{1}\right)$;

(iii) $\Phi_{h}\left(x ; z_{0} ; z_{1}\right)$ for any $x \in X^{r}$ is a homogeneous function of degree 1 of $\left(z_{0} ; z_{1}\right)$.

It follows that for $t_{1} \geqq 0, t_{2} \geqq 0$ we have

$$
\Phi_{h}\left(x ; t_{1} z_{0}^{(\mathbf{1})}+t_{2} z_{0}^{(2)} ; t_{1} z_{1}^{(1)}+t_{2} z_{1}^{(2)}\right) \leqq t_{1} \Phi_{h}\left(x ; z_{0}^{(1)} ; z_{1}^{(1)}\right)+t_{2} \Phi_{h}\left(x ; z_{0}^{(2)} ; z_{1}^{(2)}\right)
$$

and moreover

$$
\Phi_{h}(x ; 0 ; 0)=0 .
$$

We assume similar hypotheses on the $\Psi_{h}(x ; z)$ :

(i) $\Psi_{h}(x ; z)$ is a continuous function of $(x ; z)$ when $x$ varies in each $(r-1)$ cell of $\partial A$ and $z$ is an arbitrary $r$-vector;

(ii) $\Psi_{h}(x ; z)$ for any $x \in \partial A$ is a convex function of $z$;

(iii) $\Psi_{h}(x ; z)$ for any $x \in \partial A$ is a homogeneous function of degree 1 of $z$.

We have for $t_{1} \geqq 0, t_{2} \geqq 0$

$$
\Psi_{h}\left(x, t_{1} z^{(1)}+t_{2} z^{(2)}\right) \leqq t_{1} \Psi\left(x, z^{(1)}\right)+t_{2} \Psi\left(x, z^{(2)}\right) \quad \Psi(x, 0)=0 .
$$

Let $H$ be the space $H_{1}(A)$, and let $\Sigma$ be the subset of $\partial A$ considered in stating the Signorini problem in Sect. 5.

Let $A_{h}(h=1, \ldots, l)$ be a subdomain of $A$ and $\Sigma_{h}$ a subset of $\Sigma$ composed by $(r-1)$-cells of $\partial A$.

We shall define the closed convex set $V$ as the set of all the functions of $H_{1}(A)$ such that

$$
\begin{aligned}
\Phi_{h}\left(x, v ; v_{/ 1}, \ldots, v_{/ r}\right) \leqq 0 & \text { a.e. in } A_{h}(h=1, \ldots, l), \\
\Psi_{h}(x, \tau v) \leqq 0 & \text { a.e. on } \Sigma_{h}\left(h=1, \ldots, l^{\prime}\right) .
\end{aligned}
$$

Let $B(u, v)$ be the bilinear form of elasticity already considered in Sect. 5 . We have

$$
B(v, v) \geqq c_{1} \int_{A}\left(v_{i / j}+v_{j / i}\right)\left(v_{i / j}+v_{j / i}\right) d x \quad\left(c_{1}>0\right) .
$$

The kernel of $B(v, v)$ is the space $R$ of the rigid displacements. Let $Q$ be the projector of $H_{1}(A)$ onto $R$. For any vector $v \in H_{1}(A)$ such that $Q v=0$, we have, as it is easily seen,

$$
\int_{A} v d x=0, \quad \int_{A}\left(v_{i / j}-v_{j / i}\right) d x=0 .
$$

Then, if we assume that Korn's second inequality holds in $A,^{\mathbf{1 4}}$ from the remark contained in Sect. 12 of E.T.E. and from the footnote ${ }^{17}$ of that paper it follows that $B(v, v)$ satisfies Hypothesis (I) of Sect. 1. Since $R$ is finite dimensional, Hypothesis (II) is satisfied too.

Let us now consider the subset $R^{\prime}=R \cap V$. Let $R^{*}$ be the subset of $R^{\prime}$ formed by all the vectors of $R^{\prime}$ which are bilateral, i.e. $R^{*}$ is defined as follows

$$
R^{*} \equiv\left\{\varrho ; \varrho \in R^{\prime}, \varrho \in R \Rightarrow-\varrho \in R\right\} .
$$

14 Actually from the hypotheses assumed on $A$, it is possible to prove that $A$ satisfies a restricted cone hypothesis. 
It is easy to prove that $R^{*}$ is a linear space. Let

$$
f \in H_{0}(A), \quad g \in \mathscr{L}^{2}\left(\Sigma^{*}\right) \quad\left[\Sigma^{*}=\partial A-\Sigma\right] .
$$

By the generalized Signorini problem we shall mean the following unilateral problem

$$
B(u, v-u) \geqq \int_{A} f(v-u) d x+\int_{\Sigma^{*}} g(v-u) d \sigma, \quad u \in V, \forall v \in V .
$$

It is equivalent to minimize

$$
\mathscr{I}(v)=\frac{1}{2} B(v, v)-\int_{A} f v d x-\int_{\Sigma^{*}} g v d \sigma
$$

in $V$. The Signorini problem is a particular case of this more general problem, as is easily seen.

7.I If for every $\varrho \in R^{\prime}$

$$
F(\varrho) \equiv \int_{A} f \varrho d x+\int_{\Sigma^{*}} g \varrho d \sigma \leqq 0
$$

and if the sign $=$ holds when and only when $\varrho \in R^{*}$, the generalized Signorini problem has a solution. If $u$ is a solution of (7.5), any other solution $u^{\prime}$ is given by $u^{\prime}=u+\varrho$, where $\varrho$ is a rigid displacement such that $F(\varrho)=0, u+\varrho \in V$.

We may apply Theorem 1.II, assuming $u_{0}=0$. We need only to show that, if $\widetilde{Q}$ is the projector of $H_{1}(A)$ onto $R^{*}$ and $\widetilde{P}=I-\widetilde{Q}$, then $\widetilde{P}[V]$ is closed. If $v \in V$, from (7.1) we have

$$
\begin{aligned}
\Phi_{h}\left(x ; \widetilde{P} v ;(\widetilde{P} v)_{/ 1}, \ldots,(\widetilde{P} v)_{l_{r}}\right) & \leqq \Phi_{h}\left(x ; v ; v_{/ 1}, \ldots, v_{i_{r}}\right) \\
& +\Phi_{h}\left(x ;-\widetilde{Q} v ;(-\widetilde{Q} v)_{/ 1}, \ldots,(-\widetilde{Q} v)_{/ r}\right) \leqq 0 \quad \text { a.e. in } A_{h}(h=1, \ldots, l)
\end{aligned}
$$

and, analogously, from (7.2) we deduce that

$$
\Psi_{h}(x ; \tau \widetilde{P} v) \leqq 0 \quad \text { a.e. on } \Sigma_{h}\left(h=1, \ldots, l^{\prime}\right) .
$$

This means $\widetilde{P}[V]<V$. Hence $\widetilde{P}[V]$ is closed. The statement about uniqueness follows from 1.VII.

Let us explicitly note the following particular case of Theorem 1.IV. Let $P=I-Q$.

7.II If for every $w \in P\left[H_{1}(A)\right]$ there exists a $\varrho \in R$ such that $w+\varrho \in V$, and if $F(\varrho)=0$ for every $\varrho \in R$, there exists a solution of the unilateral problem (7.5).

8. Regularization theorem: interior regularity. We consider now a regularity theorem which, under suitable hypotheses, is able to guarantee a certain regularity to a solution of a unilateral problem in the neighborhood of an interior point $x^{0}$ of $A$. We consider a bilinear form of the following kind

$$
B(u, v)=\int_{A}\left\{\alpha_{h k}(x) u_{/ h} v_{/ k}+\beta_{h}(x) u_{/ h} v+\beta_{h}^{\prime}(x) u v_{/ h}+\gamma(x) u v\right\} d x,
$$

where $A$ is a bounded domain of $X^{r}$, and assume that the $n \times n$ matrices $\alpha_{h k}$ belong to $C^{2}(\bar{A})$, the matrices $\beta_{h}^{\prime}$ belong to $C^{1}(\bar{A})^{15}$ and the matrices $\beta_{h}, \gamma$ are bounded and measurable in $A$. Moreover

$$
\alpha_{h k}(x) \equiv \bar{\alpha}_{k h}(x) .
$$

15 Actually we shall see that the theorem, we are going to prove, still holds, with the same proof, under less restrictive assumptions on these matrices. 
Let us suppose that there exists a solution $u$ of the unilateral problem

$$
B(u, v-u) \geqq F(v-u), \quad u \in V, \forall v \in V,
$$

that $V$ is a closed convex set of the space $H_{1}(A)$ (of the $n$-vector valued functions) and that $F(v)$ is a bounded linear functional in $H_{1}(A)$ such that, for every $v \in \stackrel{\circ}{H}_{1}(A)$,

with $f \in H_{0}(A)$.

$$
F(v)=\int_{A} f v d x
$$

Let us assume the following hypotheses:

1) If $v$ is any function of $V$ and $\varphi$ a nonnegative scalar function of $C^{\infty}$, the function $\varphi v$ belongs to $V$;

2) There exists a subdomain $E$ of $A$ such that

$$
c\|v\|^{2} \leqq B(v, v), \quad \forall v \in \stackrel{\circ}{H}_{1}(E), \quad(c>0) .
$$

3) Let $v \in V$ and $s p t v \in E$. Let $y$ be such that $s p t v(x+y)<E$. Then $v(x+y) \in V$.

Observe that these conditions are satisfied in the generalized Signorini problem if we assume the further hypothesis that the functions $\Phi_{h}$ do not depend on $x$ and on $z_{1}$ but are functions of the variable $z_{0}$ alone.

8.I The solution $u$ of the unilateral problem (8.2) belongs to $H_{2}(I)$ for any $I$ such that $\bar{I} \subset E$. Given $x_{0} \in E$, there exists a $\delta>0$ such that $\overline{\Gamma_{\delta}} \in E$ and

$$
\|u\|_{2, \Gamma_{\delta}}^{2} \leqq c_{0}\left(\|f\|_{0, \Gamma_{2} \delta}^{2}+\|u\|_{1, \Gamma_{2 \delta}}^{2}\right)
$$

where $\Gamma_{\varrho}$ is the ball of center $x^{0} \in E$ and radius $\varrho$ and where $c_{0}$ is a positive constant depending only on the coefficients of the bilinear form (8.1) and on $x^{0}$.

Let us first remark that, because of the hypotheses we have assumed on $V$, we have $B(u, u)=F(u), B(u, v) \geqq F(v)(\forall v \in V)$. If $\psi$ is a scalar function of $C^{\infty}$ such that $0 \leqq \psi \leqq 1$, we have $B(u, \psi u) \geqq F(\psi u), B(u,(1-\psi) u) \geqq F(u)-F(\psi u)$. Hence $B(u, \psi u)=F(\psi u)$. Fixed $x^{0} \in E$, let $\delta>0$ be such that $\bar{\Gamma}_{2 \delta} \in E$. Let $\varphi(x)$ be a scalar function of $C^{\infty}$ such that $\varphi(x) \geqq 0, \varphi(x) \equiv 1$ for $\left|x-x_{0}\right| \leqq \delta, \varphi(x) \equiv 0$ for $\left|x-x_{0}\right| \geqq(3 / 2) \delta$. Set $U(x)=\varphi(x) u(x)$. Using arguments employed in E.T.E., it is easy to see that for $0<|y| \leqq \delta / 2$

$$
\left\|\frac{2 U(x)-U(x-y)-U(x+y)}{|y|^{2}}\right\|_{0, A} \leqq c_{1}\left\|\frac{U(x+y)-U(x)}{|y|}\right\|_{1, A} \quad\left(c_{1}>0\right) .
$$

Moreover, if $g \in C^{2}(\bar{A})$

$$
\begin{array}{r}
\left\|\frac{2 g(x) U(x)-g(x-y) U(x-y)-g(x+y) U(x+y)}{|y|^{2}}\right\|_{0, A} \\
\leqq c_{2}\left\|\frac{2 U(x)-U(x-y)-U(x+y)}{|y|^{2}}\right\|_{0, A}
\end{array}
$$

where $c_{2}$ depends on the $C^{2}$ norm of $g(x)$.

Let us recall that, if the function of $y$

$$
\left\|\frac{U(x+y)-U(x)}{|y|}\right\|_{1, A}
$$


is bounded by $c_{3}$, then $\|U\|_{2, A}$ is finite and $\|U\|_{2, A} \leqq c_{4} c_{3}$. We have for $0 \leqq|y| \leqq \delta / 2$ $B[U(x+y)-U(x), U(x+y)-U(x)]=2 B[u(x), \varphi(x) U(x)]$

$$
\begin{aligned}
& -2 B[u(x), \varphi(x) U(x-y)] \\
& +\int_{A}\left\{\alpha_{h k}(x)\left[U_{\mid h}(x+y)-U_{\mid h}(x)\right]\left[U_{\mid k}(x+y)-U_{\mid k}(x)\right]\right. \\
& -2 \alpha_{h k}(x) u_{/ h}(x)[\varphi(x) U(x)]_{/ k}+2 \alpha_{h k}(x) u_{/ h}(x)[\varphi(x) U(x-y)]_{/ k} \\
& +\beta_{h}(x)\left[U_{\mid h}(x+y)-U_{\mid h}(x)\right][U(x+y)-U(x)] \\
& -2 \beta_{h}(x) u_{l h}(x) \varphi(x) U(x)+2 \beta_{h}(x) u_{/ h}(x) \varphi(x) U(x-y) \\
& +\beta_{h}^{\prime}(x)[U(x+y)-U(x)]\left[U_{\mid h}(x+y) U_{\mid h}(x)\right] \\
& -2 \beta_{h}^{\prime}(x) u(x)[\varphi(x) U(x)]_{/ h}+2 \beta_{h}^{\prime}(x) u(x)[\varphi(x) U(x-y)]_{/ h} \\
& +\gamma(x)[U(x+y)-U(x)][U(x+y)-U(x)] \\
& -2 \gamma(x) U(x) U(x)+2 \gamma(x) U(x) U(x-y)\} d x .
\end{aligned}
$$

Set

$$
\lambda(\gamma)=B[U(x+y)-U(x), U(x+y)-U(x)] .
$$

From (8.3), keeping in mind that

$$
\begin{aligned}
B\left[u(x), \varphi^{2}(x) u(x)\right]= & (\varphi(x) f(x), U(x))_{0} \\
& -B[u(x), \varphi(x) \varphi(x-y) u(x-y)] \leqq-(\varphi(x) f(x), U(x-y))_{0},
\end{aligned}
$$

we deduce an inequality which we write briefly as $\lambda(y) \leqq \mu(y)$. On the other hand we have $\lambda(y)=\lambda(-y)+\sigma(y)$, where

$$
\begin{aligned}
\sigma(y)= & \int_{A}\left\{\left[\alpha_{h k}(x-y)-\alpha_{h k}(x)\right][U(x-y)-U(x)]_{/ h}[U(x-y)-U(x)]_{/ k}\right. \\
& +\left[\beta_{h}(x-y)-\beta_{h}(x)\right][U(x-y)-U(x)]_{/ h}[U(x-y)-U(x)] \\
& +\left[\beta_{h}^{\prime}(x-y)-\beta_{h}^{\prime}(x)\right][U(x-y)-U(x)][U(x-y)-U(x)]_{/ h} \\
& +[\gamma(x-y)-\gamma(x)][U(x-y)-U(x)][U(x-y)-U(x)]\} d x .
\end{aligned}
$$

Hence $\lambda(y) \leqq 2^{-1}[\mu(y)+\mu(-y)+\sigma(y)]$. If we set $\Phi(x)=\varphi(x) f(x)$, this last inequality, after simple transformations, gives

$$
\begin{aligned}
& B[U(x+y)-U(x), U(x+y)-U(x)] \leqq(\Phi(x), 2 U(x)-U(x-y)-U(x+y))_{0} \\
& +\int_{A}\left\{\left[\varphi_{\mid h}(x+y) u(x+y)-\varphi_{\mid h}(x) u(x)\right]\left[\bar{\alpha}_{h k}(x+y) U_{\mid k}(x+y)-\bar{\alpha}_{h k}(x) U_{\mid k}(x)\right]\right. \\
& +\varphi_{\mid k}(x) u_{/ k}(x)\left[\bar{\alpha}_{h k}(x-y) U(x-y)+\bar{\alpha}_{h k}(x+y) U(x+y)-2 \bar{\alpha}_{h k}(x) U(x)\right] \\
& -\left[\alpha_{h k}(x-y)-\alpha_{h k}(x)\right] u_{/ h}(x)\left([\varphi(x) U(x-y)]_{/ k}-[\varphi(x) U(x)]_{/ k}\right) \\
& -\left[\alpha_{h k}(x+y)-\alpha_{h k}(x)\right] u_{l k}(x)\left([\varphi(x) U(x+y)]_{/ k}-[\varphi(x) U(x)]_{/ k}\right) \\
& -\left[\alpha_{h k}(x-y)+\alpha_{h k}(x+y)-2 \alpha_{h_{k}}(x)\right] u_{l h}(x)[\varphi(x) U(x)]_{/ k} \\
& +2^{-1}\left[\alpha_{h k}(x-y)+\alpha_{h k}(x+y)-2 \alpha_{h k}(x)\right] U_{l h}(x) U_{l k}(x) \\
& +2^{-1} \beta_{h}(x)\left[U_{l h}(x+y)-U_{l h}(x)\right][U(x+y)-U(x)] \\
& +2^{-1} \beta_{h}(x)\left[U_{l h}(x-y)-U_{\mid h}(x)\right][U(x-y)-U(x)] \\
& +\beta_{h}(x) \varphi(x) u_{l h}(x)[U(x-y)+U(x+y)-2 U(x)] \\
& +2^{-1} \beta_{h}^{\prime}(x)[U(x+y)-U(x)]\left[U_{l h}(x+y)-U_{\mid h}(x)\right] \\
& +2^{-1} \beta_{h}^{\prime}(x)[U(x-y)-U(x)]\left[U_{l h}(x-y)-U_{\mid h}(x)\right] \\
& -\left[\beta_{h}^{\prime}(x+y) U(x+y)-\beta_{h}^{\prime}(x) U(x)\right]\left[U_{\mid h}(x+y)-U_{\mid h}(x)\right] \\
& +\beta_{h}^{\prime}(x) \varphi_{l h}(x) u(x)[U(x-y)+U(x+y)-2 U(x)] \\
& +2^{-1} \gamma(x)[U(x+y)-U(x)][U(x+y)-U(x)] \\
& +2^{-1} \gamma(x)[U(x-y)-U(x)][U(x-y)-U(x)] \\
& +\gamma(x) U(x)[U(x-y)+U(x+y)-2 U(x)]\} d x+2^{-1} \sigma(y) \text {. }
\end{aligned}
$$


Denoting by $p_{1}, p_{2}, \ldots$ positive constants and by \|\| norms over $A$, we have for $0<|y| \leqq \delta / 2$

$$
\begin{aligned}
c\left\|\frac{U(x+y)-U(x)}{|y|}\right\|_{1}^{2} \leqq & p_{1}\|\Phi\|\left\|\frac{U(x+y)-U(x)}{|y|}\right\|_{1} \\
& +p_{2}\|u\|_{1, \Gamma_{2} \delta}\left\|\frac{U(x+y)-U(x)}{|y|}\right\|_{1}+p_{3}\|u\|_{1, \Gamma_{2} \delta}^{2} \\
& +\sum_{h, k}^{1, r} \sup _{x, \xi \in \Gamma_{\mathbf{2}} \delta}\left|a_{h k}(x)-a_{h k}(\xi)\right|\left\|\frac{U(x+y)-U(x)}{|y|}\right\|_{1}^{2} .
\end{aligned}
$$

We can choose $\delta$ such that

$$
\sum_{h, k}^{1, r} \sup _{x, \xi \in \Gamma_{2 \delta}}\left|a_{h k}(x)-a_{h k}(\xi)\right|<c .
$$

Then the proof of the theorem is an obvious consequence of (8.4).

9. Regularization theorem: regularity near the boundary. The technique used in the preceding section can be extended so as to get analogous results concerning regularization near the regular points of the boundary of $A$.

We consider the bilinear form $B(u, v)$ given by $(8.1)$ and impose on the matrices $\alpha_{h k}, \beta_{h}, \beta_{h}^{\prime}, \gamma_{h}$ the hypotheses of Sect. 8.

Let $x^{0}$ be a point of $\partial A$, and suppose that $A$ is $C^{3}$-smooth in $x^{0}$. This means that there exists a neighborhood $I$ of $x^{0}$ such that the set $J=\bar{I} \cap \bar{A}$ can be mapped $C^{3}$-homeomorphically onto the closed semiball $\bar{\Sigma}^{+}:|y|^{2}+t^{2} \leqq 1, t \geqq 0$ of the $r$-dimensional $(y, t)$-space in such a way that the set $\bar{I} \cap \partial A$ is mapped onto the $(r-1)$-dimensional ball $t=0,|y| \leqq 1$.

As in Sect. 8, we assume that there exists a solution $u$ of the unilateral problem (8.2). We suppose that the functional $F(v)$ for every $v \in H_{1}(A)$ such that $s p t v \in J$, admits the following representation

with $f \in H_{0}(A)$.

$$
F(v)=\int_{A} f v d x
$$

We now assume the following hypotheses:

1) If $v$ is any function of $V$ and $\varphi$ a nonnegative scalar function of $C^{\infty}$, the function $\varphi v$ belongs to $V$.

2) We have

$$
c\|v\|^{2} \leqq B(v, v), \quad \forall v \in H_{1}(A), s p t v \in J \quad(c>0) .
$$

3) Let $x=x(\xi) \equiv x(y, t)$ the $C^{3}$-homeomorphism from $\bar{\Sigma}^{+}$to $J$. Let $E$ be the image of the semiball $\Sigma^{+}:|y|^{2}+t^{2}<1, t \geqq 0$ under this homeomorphism. Let $v \in V$ and $s p t v \in E$. Let $y$ be such that $s p t v[x(\xi+y)]<\Sigma^{+}$. Then $v[x(\xi+y)] \in V$.

4) The solution $u$ of the unilateral problem (8.2) satisfies in $A \cap I$ the differential equation

$$
\left(\alpha_{h k} u_{/ h}\right)_{/ k}+\beta_{h} u_{/ h}-\left(\beta_{h}^{\prime} u\right)_{h h}+\gamma u+f=0 .
$$

If $U$ is a subset of $\bar{\Sigma}^{+}$we shall denote by $x(U)$ its image under the homeomorphism $x=x(\xi)$.

9.I There exists $\delta>0$ such that $\Gamma_{\delta}^{+} \subset \Sigma^{+}, u \in H_{2}\left[x\left(\Gamma_{\delta}^{+}\right)\right]$and

$$
\|u\|_{2, x\left(\Gamma_{\delta}^{+}\right)}^{2} \leqq c_{0}\left(\|f\|_{0, x\left(\Gamma_{2 \delta}^{+} \delta\right)}^{2}+\|u\|_{1, x\left(\Gamma_{2 \delta}^{+}\right)}^{2}\right) ;
$$


$\Gamma_{\delta}^{+} i$ s the semiball $|y|^{2}+t^{2} \leqq \delta^{2}, t>0$ and $c_{0}$ a positive constant only depending on the coefficients of the bilinear form (8.1) and on $x^{0}$.

The proof parallels the one of Theorem 8.I. We have first to transform the bilinear form $B(u, v)$, for any $v$ such that $s p t v \in E$, by using the homeomorphism $x=x(\xi)$. We get a bilinear form which we indicate as follows

$$
\widetilde{B}(u, v)=\int_{\Sigma^{+}}\left(\tilde{\alpha}_{h k} u_{/ h} v_{/ k}+\tilde{\beta}_{h} u_{i h} v+\tilde{\beta}_{h}^{\prime} u v_{/ h}+\tilde{\gamma} u v\right) d \xi .
$$

From now on we exactly follow the proof of Theorem 8.I with the only difference that now $y$ must be parallel to the hyperplane $t=0$. Thus we arrive at inequality (8.4). However the boundedness of

$$
\left\|\frac{U(x+y)-U(x)}{|y|}\right\|_{1}^{2}
$$

is only able, in this case, to insure that all second derivatives of $U$ except $U_{t t}$ belong to $\mathscr{L}^{2}\left(\Sigma^{+}\right)$. Since $u$ satisfies the differential equation (9.1), after writing this equation in the variables $y, t$, we deduce, since, for Hypothesis 2 ), the equation is elliptic, that $U_{t t}=\mathscr{L}^{2}\left(\Sigma^{+}\right)$and, in consequence, the proof of the theorem.

Remark. In the proof of Theorem 8.I we considered in the integrand of the bilinear form (8.1) the presence of the term $\beta_{h}^{\prime} u v_{/ h}$. This could have been avoided by an integration by parts. However since this term cannot be eliminated in the proof of Theorem 9.I, we have retained it also in the proof of the earlier theorem.

In the case of the generalized Signorini problem, assuming that $\Phi_{h}$ does not depend on $z_{i}$, the hypotheses assumed in this section are satisfied if we suppose that: i) the domains $A_{h}(h=1, \ldots, l)$ (see Sect. 7) are all disjoint from $J$; ii) for each $h\left(h=1, \ldots, l^{\prime}\right)$ either the subset $\Sigma_{h}$ is disjoint from $I \cap \partial A$ or the function $\Psi_{h}(x, z)$ does not depend on $x$ (for $x \in \bar{I} \cap \partial A$ ).

In the original Signorini problem there is no question about the $\Phi_{h}$ 's since $l=0$; the condition ii) concerning the $\Psi_{h}^{\prime}$ s is not satisfied if $x^{0} \in \Sigma$ since $l^{\prime}=1$, $\Psi_{1}=-v(x) z, \Sigma_{1} \equiv \Sigma$. However, we may overcome this difficulty by introducing in the set $E$ an orthogonal system of unit-vectors $v_{1}(x), \ldots, v_{r}(x)$ such that: 1) $v_{i}(x) \in C^{2}(E)(i=1, \ldots, r)$; 2) $v_{r}(x) \equiv v(x)$ for $x \in I \cap \partial A$. If $\partial A$ is $C^{3}$-smooth in $x^{0}$ the construction of this set of vectors is trivial. For each $v \in H_{1}(A)$ and for $x \in E$, set $v(x)=\bar{v}_{i}(x) v_{i}(x)$. Let

$$
a_{h k} \equiv\left\{a_{h k}^{i j}\right\} \equiv\left\{a_{j h, i k}\right\} \quad(i, j=1, \ldots, r)
$$

be the matrices considered in Sect. 12 of E.T.E. in connection with problems of elasticity and employed in Sect. 5.16 If $s p t v \in E$ we have

$$
a_{h k} u_{/ h} v_{/ k}=a_{h k} v_{i} \tilde{u}_{i / h} v_{j} \tilde{v}_{j / k}+a_{h k} v_{i} \tilde{u}_{i / h} v_{j / k} \tilde{v}_{j}+a_{h k} v_{i / h} \tilde{u}_{i} v_{j} \tilde{v}_{j / k}+a_{h k} v_{i / h} \tilde{u}_{i} v_{j / k} \tilde{u}_{j} \text {. }
$$

Let us define the matrices $\alpha_{h k}, \beta_{h}, \beta_{h}^{\prime}, \gamma$ as follows:

$$
\alpha_{h k} \equiv\left\{a_{h k} v_{i} v_{j}\right\}, \quad \beta_{h} \equiv\left\{a_{h k} v_{i} v_{j / k}\right\}, \quad \beta_{h}^{\prime} \equiv\left\{a_{k h} v_{i / k} v_{j}\right\}, \quad \gamma \equiv\left\{a_{h k} v_{i / h} v_{j / k}\right\} .
$$

${ }^{16}$ By writing $\left\{a_{h k}^{i j}\right\}$ we mean the matrix $a_{h k}$ which has $a_{h k}^{i j}$ as entry in the $i$-th row and in the $j$-th column. Thus we assume $a_{h k}^{i j}=a_{j h}, i_{k}$. 
If we denote by $\tilde{u}$ and $\tilde{v}$ the $r$-vectors having as components $\tilde{u}_{1}, \ldots, \tilde{u}_{r}$ and $\tilde{v}_{1}, \ldots, \tilde{v}_{r}$, we have, if $s p t v \in E$,

$$
B(u, v)=\int_{A}\left(\alpha_{h k} u_{/ h} v_{/ k}+\beta_{h} u_{/ h} v+\beta_{h}^{\prime} u v_{/ h}+\gamma u v\right) d x
$$

and the constraint of the Signorini problem is expressed by $-\tilde{v}_{\boldsymbol{r}}(x) \leqq 0$ $(x \in \bar{I} \cap \partial A)$. Then the above condition ii) is satisfied and we can apply the regularization theory developed in this section.

10. Analysis of the Signorini problem. On the regular bounded domain $A$ we shall assume the following more specific hypotheses:

1) $\partial A$ is decomposable into a finite set of non-overlapping differentiable $(r-1)$-cells of class $C^{\infty}: \Gamma_{1}, \ldots, \Gamma_{q}$.

2) It is possible to define a unit vector $\mu(x)$ which is a function of the point $x$ variable on $\partial A$, which is a continuous function of $x$ on $\partial A$ and which always points inside $A$, while $-\mu(x)$ points into the complement of $\bar{A}$. Denoting by $\omega$ the angle (between 0 and $\pi$ ) which $\mu(x)$ forms with the inner unit normal vector $v(x)$, we always have $0 \leqq \omega \leqq \omega_{0}<\pi / 2$ in every point $x$ of $\Gamma_{k}-\partial \Gamma_{k}(k=1, \ldots, q$; $\partial \Gamma_{k} \equiv$ border of $\Gamma_{k}$ ).

3) Denoting by $x=x^{k}(t)$ the parametric representation of $\Gamma_{k}$ on $T^{r-1}$ (see Footnote ${ }^{7}$, p. 402) we suppose that $\mu\left[x^{k}(t)\right] \in C^{\infty}\left(T^{r-1}\right)$.

4) There exists a positive number $\lambda_{0}$ such that for every $\lambda$ such that $0<\lambda \leqq \lambda_{0}$ the range described by $y=x+\lambda \mu(x)$ as $x$ varies on $\partial A$ is entirely contained in $A$ and is in one-to-one correspondence with $\partial A$.

It is easy to prove that conditions 1), 2), 3), 4), imply that $A$ is properly regular.

We shall consider in the domain $A$ the Signorini problem. Assume

Let

$$
\Sigma=\Gamma_{1} \cup \Gamma_{2} \cup \ldots \cup \Gamma_{q^{\prime}} \quad\left(q^{\prime} \leqq q\right) .
$$

$$
f \in H_{0}(A), \quad g \in \mathscr{L}^{2}\left(\Sigma^{*}\right) \quad\left(\Sigma^{*}=\partial A-\Sigma\right) .
$$

From the theory we have developed in the preceding sections we derive this theorem

10.I Set

$$
B(u, v)=\int_{A} a_{h k}(x) u_{i h} v_{i k} d x, \quad F(v)=\int_{A} f v d x+\int_{\Sigma^{*}} g v d \sigma,
$$

where $\left\{a_{h k}^{i j}\right\} \equiv\left\{a_{j h, i k}\right\}$ are the matrices of elasticity (see E.T.E., Sect. 12). Let $V$ be the convex set formed by all the functions of $H_{1}(A)$ such that $v(x) v(x) \geqq 0$ on $\partial A .^{17}$ Let $R^{\prime}=R \cap V$ and $R^{*}$ be the subset of $R^{\prime}$ formed by all the bilateral displacements. $A$ necessary condition for the existence of a minimum of the functional $\mathscr{I}(v)=$ $2^{-1} B(v, v)-F(v)$ in $V$ is

$$
F(\varrho) \leqq 0 \quad \forall \varrho \in R^{\prime} .
$$

If this condition is satisfied in the strong sense, i.e. if the sign=holds when and only when $\varrho \in R^{*}$, then $\mathscr{I}(v)$ has an absolute minimum in $V$. If $u$ is a minimizing function, $u$ is a solution in $A$ of the differential system

$$
\left(a_{h k} u_{h h}\right)_{h k}+f=0 \text {. }
$$

17 Of course the inequality $v(x) v(x) \geqq 0$ must be understood in the sense of the functions of $H_{1}(A)$, i.e. almost everywhere. 
If $f \in C^{\infty}(A)$, then $u \in C^{\infty}(A)$. If $q^{\prime}<q, \quad x^{0} \in \Gamma_{k}-\partial \Gamma_{k} \quad\left(k=q^{\prime}+1, \ldots, q\right)$ and $g\left[x^{k}(x)\right] \in C^{\infty}\left(T^{r-1}\right)$. Then $u \in C^{\infty}(\bar{B})$, for any domain $B$ such that $\bar{B} C \bar{A} \cap(A \cup I)$, where $I$ is a suitable neighborhood of $x^{0},{ }^{18}$ and $u$ satisfies in $\partial A \cap I$ the boundary condition

$$
\sigma_{i h}(u) v_{h}=g_{i} \quad\left[\sigma_{i h}(u)=-a_{i h, j k} u_{j \mid k}\right] .
$$

If $x^{0} \in \Gamma_{k}-\partial \Gamma_{k}\left(k=1, \ldots, q^{\prime}\right)$ then $u \in H_{2}(A \cap I),{ }^{19}$ where $I$ is a suitable neighborhood of $x^{0}$, and $u$ satisfies in $\partial A \cap I$ the "ambiguous boundary conditions"

$$
\text { either }\left\{\begin{array} { l } 
{ u _ { i } v _ { i } = 0 } \\
{ \sigma _ { i h } ( u ) v _ { i } v _ { h } \geqq 0 } \\
{ \sigma _ { i h } ( u ) v _ { i } \tau _ { h } = 0 }
\end{array} \quad \text { (10.3) } \quad \text { or } \left\{\begin{array}{l}
u_{i} v_{i}>0 \\
\sigma_{i h}(u) v_{i} v_{h}=0 \\
\sigma_{i h}(u) v_{i} \tau_{h}=0
\end{array}\right.\right.
$$

where $\tau$ is any vector tangent to $\Sigma$ in the points of $\partial A \cap I$.

The conditions (10.3) express the fact that at the point of $\partial A \cap I$ under consideration the elastic body in its equilibrium configuration rests on $\Sigma$, and therefore, that the reaction of the constraints has a nonnegative component along the inward normal. Any tangential component of such a reaction is null since the surface $\Sigma$ is supposed frictionless. On the other hand, if the conditions (10.4) are satisfied, this means that in coming to equilibrium the body has left the supporting surface, which therefore no longer reacts on the body.

Evidently it is not known a priori which of the two sets of conditions (10.3) and (10.4) is to be satisfied at a given point of $\Sigma$. Hence the use of the name proposed by Signorini of "ambiguous boundary conditions".

All the facts considered in the statement of the theorem are evident consequences of the theory developed in the preceding sections. For proving that the ambiguous conditions (10.3) (10.4) are satisfied in $\partial A \cap I$ ( $I$ being a suitable neighborhood of a regular point $x^{0}$ of $\Sigma$ ), one only needs to repeat, with easy generalizations, the arguments used in the example $I$ of Sect. 6 . Thus one shows that, almost everywhere on $\partial A \cap I$, the following conditions are satisfied:

$$
u_{i} \nu_{i} \geqq 0, \quad \sigma_{i k} v_{i} v_{k} \geqq 0, \quad \sigma_{i k} \nu_{i} v_{k} u_{j} \nu_{j}=0, \quad \sigma_{i k} \nu_{i} \tau_{k}=0 .
$$

Since the functions $u_{i}, \sigma_{i k}$ are defined almost everywhere, we may assume, by suitably defining these functions on sets of measure zero, ${ }^{20}$ that (10.5) hold at every point of $\partial A \cap I$. Thus we deduce that in $\partial A \cap I$ the ambiguous conditions (10.3) (10.4) hold.

The following theorem gives information about the global nature of the reaction that the supporting surface $\Sigma$ exerts on the elastic body.

10.II $A$ real-valued nonnegative measure $\gamma(B)$ is defined in the $\sigma$-ring $\{B\}_{\Sigma}$ of the Borel sets $B$ contained in $\Sigma$ such that, if $u$ minimizes $\mathscr{I}(v)$ in $V$, we have for every $v \in H_{1}(A) \cap C^{0}(A \cup \Sigma)$

$$
\int_{\Sigma} v_{i} v_{i} d \gamma=B(u, v)-F(v) .
$$

18 Actually, in the case when $g \equiv 0$, this is consequence of Theorem 3.III. For the general case we refer the reader to the paper [6] (see Bibliography of E.T.E.).

19 In the case $r \leqq 3$ this implies that $u$ is continuous in $\overline{A \cup I}$ (see E.T.E., 2.VI).

20 Actually, in the case $r \leqq 3$, we have to take care only of the functions $\sigma_{i k}$. 
The singular set of the measure $\gamma$ is contained in

$$
\bigcup_{k=1}^{q^{\prime}} \partial \Gamma_{k}
$$

and the Lebesgue derivative of $\gamma$ is the function $\sigma_{i h}(u) \nu_{i} \nu_{h}{ }^{21}$

We denote by $W$ the linear manifold that consists of the real valued functions $w$ which are defined almost everywhere on $\Sigma$ and are such that, for every $w$, and almost everywhere on $\Sigma$, we have $w=v_{i} v_{i}$ with $v \in H_{1}(A) \cap C^{0}(A \cup \Sigma)$. We put

$$
\Phi(w)=B(u, v)-F(v) .
$$

The linear functional $\Phi$ is defined without ambiguity by (10.7) for every $w \in W$, because, if $\tilde{v} \in H_{1}(A) \cap C^{0}(A \cup \Sigma)$ and $w=\tilde{v}_{i} v_{i}$ a.e. on $\Sigma$, then, since $v-\tilde{v}$ is a bilateral displacement of $V$ (i.e. both $v-\tilde{v}$ and $\tilde{v}-v$ belong to $V$ ), we have $B(u, v)-F(v)=B(u, \tilde{v})-F(\tilde{v})$.

Let $A_{\lambda}\left(0<\lambda \leqq \lambda_{0}\right)$ be the domain bounded by the range of $x=\xi+\lambda \mu(\xi)$ as $\xi$ describes $\partial A$. Let $\psi(x)$ be a real valued function of $C^{\infty}$ which is null in $A_{\lambda_{0}}$ and is equal to 1 in the exterior of $A_{\lambda_{0} / 2}$. Set

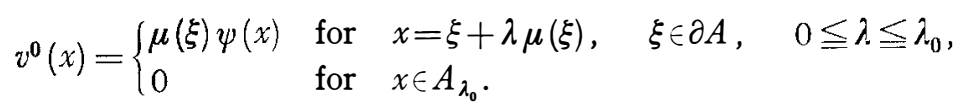

If $\lambda_{0}$ is sufficiently small the function $v^{0}(x)$ is defined everywhere in $\bar{A}$ and belongs to $H_{1}(A) \cap C^{0}(A \cup \Sigma)$. Moreover we have on $\partial A: v_{i}^{\mathbf{0}} \nu_{i} \geqq \cos \omega_{0}>0$.

We can assume that every $w \in W$ coincides in $\Gamma_{k}\left(k=1, \ldots, q^{\prime}\right)$ with a continuous function $w_{k}$. Let us use the symbol max $\left|w_{k}\right|$ for the maximum of $\left|w_{k}\right|$ in $\Gamma_{k}$ and put $\mathscr{M}(w)=\max \left(\max \left|w_{1}\right|, \ldots, \max \left|w_{q^{\prime}}\right|\right)$. Consider the Banach space $\bar{W}$ obtained by functional completion from $W$ by means of the norm $\mathscr{M}(w)$. On every $\Gamma_{k}\left(k=1, \ldots, q^{\prime}\right)$ we have for any $w \in W$

and therefore

$$
-\frac{\mathscr{A}(w)}{\cos w_{0}} v_{i}^{0}(x) v_{i}(x) \leqq w(x) \leqq \frac{\mathscr{M}(w)}{\cos w_{0}} v_{i}^{0}(x) v_{i}(x)
$$

$$
|\Phi(w)| \leqq-\frac{1}{\cos w_{0}}\left[B\left(u, v^{0}\right)-F\left(v^{0}\right)\right] \mathscr{M}(w) .
$$

Thus $\Phi$ is a continuous functional in $W$. On the other hand, when $w \in W$ and $w \geqq 0$ on $\Sigma$, we have, because of $(10.7), \Phi(w) \geqq 0$. From classical theorems of Functional Analysis we deduce the existence of the non-negative measure $\gamma$ such that (10.6) holds.

21 A $\sigma$-ring of sets is a family of sets which is closed with respect to union and intersection either of a finite or of a countable collection of sets of the family. The $\sigma$-ring $\{B\}_{\Sigma}$ is the intersection of all the $\sigma$-rings formed by subsets of $\Sigma$. A measure defined on $\{B\}_{\Sigma}$ is any real valued function of $B$ such that $\gamma(B)=\sum_{k} \gamma\left(B_{k}\right)$ if $B=B_{1} \cup B_{2} \cup \cdots \cup B_{k} \ldots$ and $B_{1}, \ldots, B_{k}, \ldots$ is either a finite or a countable collection of mutually disjoint sets of $\{B\}_{\Sigma}$. A measure $\gamma^{*}$ is said to be singular if, for any $B \in\{B\}_{\Sigma}, \gamma^{*}(B)=\gamma^{*}(B \cap N)$, where $N$ is a set of zero Lebesgue measure on $\Sigma$. Hence $\gamma^{*}(B)=0$ if $B \cap N=\phi$. For every measure $\gamma(B)$ defined on $\{B\}_{\Sigma}$ there exist, and are uniquely determined, a singular measure $\gamma^{*}(B)$ and a function $\varphi(x) \in \mathscr{L}^{1}(\Sigma)$ such that the Lebesgue decomposition holds

$$
\gamma(B)=\gamma^{*}(B)+\int_{B} \varphi(x) d \sigma .
$$

The above mentioned set $N$ is called the singular set of the measure $\gamma$ and the function $\varphi(x)$ is called the Lebesgue derivative of the measure $\gamma$. 
Let $x^{0} \in \Gamma_{k}-\partial \Gamma_{k}$ and let $I$ be a suitable small neighborhood of $x^{0}$ such that $u$ belongs to $H_{2}(A \cap I)$. Let $v$ be a function belonging to $H_{1}(A) \cap \stackrel{C}{(}(I)$. For the $w$ which corresponds to this particular $v$ we have by (10.2)

$$
\Phi(w)=B(u, v)-F(v)=-\int_{\partial A}\left(a_{h k} u_{l h} v_{k}\right) v d \sigma=\int_{\partial A}\left(v_{j} v_{j}\right)\left[\sigma_{i h}(u) v_{i} v_{h}\right] d \sigma .
$$

That implies that the singular set of $\gamma$ must be contained in $\bigcup_{k=1}^{q^{\prime}} \partial \Gamma_{k}$ and that the Lebesgue derivative of $\gamma$ is $\sigma_{i h}(u) v_{i} v_{h}$.

The mechanical meaning of the measure $\gamma$ is evident: $\gamma(B)$ represents the intensity of the global reaction exerted by the constraint of support on $\Sigma$ over the whole set $B$. This reaction may have concentrated stresses only on point-sets formed by singular points of $\Sigma$; no concentration can occur in the neighborhood of any regular point of $\Sigma . .^{2}$

We wish now to discuss another delicate question concerning the Signorini problem. We saw that the condition (10.1) is sufficient for the existence of the solution $u$ of the Signorini problem provided it is satisfied in the strong sense, i.e. the sign $=$ can occur in (10.1) when and only when $\varrho \in R^{*}$. We shall prove, considering a particular case of paramount mechanical interest, that the strong condition is necessary for the existence of the solution. That will lead us to a remarkable mechanical interpretation of this condition.

We take $r=3$ and we suppose that the supporting surface $\Sigma$ is planar and connected. We are permitted to suppose that $\Sigma$ is a bounded closed region of the plane $x_{3}=0$. Moreover we suppose that $\bar{A}-\Sigma$ is contained in the half-space $x_{3}>0$. The linear space of the rigid displacements is formed by the vectors $\varrho$ such that

$$
\varrho_{1}=a_{1}+b_{2} x_{3}-b_{3} x_{2}, \quad \varrho_{2}=a_{2}+b_{3} x_{1}-b_{1} x_{3}, \quad \varrho_{3}=a_{3}+b_{1} x_{2}-b_{2} x_{1},
$$

where $a_{1}, a_{2}, a_{3}, b_{1}, b_{2}, b_{3}$ are constants. The vector $\varrho$ belongs to $R^{\prime}$ if and only if

$$
a_{3}+b_{1} x_{2}-b_{2} x_{1} \geqq 0 \quad \text { for }\left(x_{1}, x_{2}\right) \in \Sigma .
$$

We are permitted to suppose that the $x_{3}$-axis intersects $\Sigma-\partial \Sigma$. That implies $a_{3} \geqq 0$. For any integrable real valued function $w$, set

$$
F_{h}(w)=\int_{A} f_{h} w d x+\int_{\Sigma^{*}} g_{h} w d \sigma \quad(h=1,2,3) .
$$

Condition (10.1) is equivalent to the following conditions

$$
\begin{gathered}
F_{1}(1)=F_{2}(1)=0, \quad F_{2}\left(x_{1}\right)-F_{1}\left(x_{2}\right)=0, \\
a_{3} F_{3}(1)-b_{2}\left[F_{3}\left(x_{1}\right)-F_{1}\left(x_{3}\right)\right]+b_{1}\left[F_{3}\left(x_{2}\right)-F_{2}\left(x_{3}\right)\right] \leqq 0 .
\end{gathered}
$$

The last inequality is to be taken for $a_{3}, b_{2}$ and $b_{1}$ satisfying (10.7). It follows that

$$
F_{3}(1) \leqq 0 \text {. }
$$

If the sign $=$ held, then from (10.8) (10.9) we could deduce that $F_{3}\left(x_{1}\right)-F_{1}\left(x_{3}\right)=0$, $F_{3}\left(x_{2}\right)-F_{2}\left(x_{3}\right)=0$, i.e. the system of the applied forces would be equilibrated. Then the problem has a solution, since it reduces to the classical one which consists in assigning the body forces and the surface forces everywhere on $\partial A$, on the assumption that the given surface forces vanish on $\Sigma$ (see Theorems 1.VI and 7.II).

22 This circumstance was conjectured by the soviet mathematician G. I. BARENBLATT, during a seminar that the author held at Moscow University in 1969. 
From now on we shall exclude equilibrated systems of applied forces.

Then we must have

$$
F_{3}(1)<0 \text {. }
$$

From (10.9) we deduce the relation

$$
F_{1}(1)\left[F_{3}\left(x_{2}\right)-F_{2}\left(x_{3}\right)\right]+F_{2}(1)\left[F_{1}\left(x_{3}\right)-F_{3}\left(x_{1}\right)\right]+F_{3}(1)\left[F_{2}\left(x_{1}\right)-F_{1}\left(x_{2}\right)\right]=0 .
$$

Since the vector $\left\{F_{1}(1), F_{2}(1), F_{3}(1)\right\}$ does not vanish, we know from elementary mechanics ${ }^{23}$ that the system of applied forces is equivalent to a single force orthogonal to the plane $x_{3}=0$, directed downwards and applied in any point of the central axis of the system which is the straight line $x_{1}=x_{1}^{0}, x_{2}=x_{2}^{0}$ with

$$
x_{1}^{0}=\frac{F_{3}\left(x_{1}\right)-F_{1}\left(x_{3}\right)}{F_{3}(1)}, \quad x_{2}^{0}=\frac{F_{3}\left(x_{2}\right)-F_{2}\left(x_{3}\right)}{F_{3}(1)} .
$$

We shall call the set obtained as the intersection of all the closed half-planes which contain $\Sigma$, the convex hull of $\Sigma$ and shall denote it by $K(\Sigma) . K(\Sigma)$ is obviously a closed convex set. A half-plane having as its origin the straight line $a_{3}+b_{1} x_{2}-b_{2} x_{1}=0$, with $a_{3}>0$, contains $\Sigma$ if and only if $a_{3}, b_{1}, b_{2}$ satisfy condition (10.8). Thus the last of (10.9) expresses the fact that $K(\Sigma)$ contains $\left(x_{\mathbf{1}}^{\mathbf{0}}, x_{\mathbf{2}}^{\mathbf{0}}\right)$. Vice-versa, if $K(\Sigma)$ contains $\left(x_{1}^{0}, x_{2}^{0}\right)$ and if the system of applied forces is equivalent to a single force, orthogonal to the plane $x_{3}=0$ and directed downwards, conditions (10.9) are satisfied and thus also (10.1).

We shall now prove that

10.III A necessary and sufficient condition in order that, given (10.1), it hold in the strong sense, is that the central axis of the system of applied forces meet $K(\Sigma)$ at an internal point.

If $\left(x_{1}^{0}, x_{2}^{0}\right)$ is internal to $K(\Sigma)$, it will be internal to every half-plane which contains $\Sigma$. This implies that the last of (10.9) is satisfied in the strong sense for $a_{3}, b_{1}, b_{2}$ satisfying (10.8), and $a_{3}>0$. Thus (10.1) is satisfied in the strong sense. Vice-versa, if (10.1) is satisfied and if we had $\left(x_{1}^{0}, x_{2}^{0}\right) \in \partial K(\Sigma)$, we could consider a straight line $a_{3}+b_{1} x_{2}-b_{2} x_{1}=0\left(a_{3}>0\right)$ passing through $\left(x_{1}^{0}, x_{2}^{0}\right)$ and such that one of the half-planes which admit this straight line origin (indeed the half-plane $\left.a_{3}+b_{1} x_{2}-b_{2} x_{1} \geqq 0\right)$ contains $K(\Sigma) \cdot{ }^{24}$ The rigid displacement $\varrho_{1}=b_{2} x_{3}, \varrho_{2}=-b_{1} x_{3}$, $\varrho_{3}=a_{3}+b_{1} x_{2}-b_{2} x_{1}$ belongs to $R^{\prime}$ and is unilateral. However, the sign $=$ holds in (10.1) for this displacement.

From this lemma it follows that the case in which (10.1) is satisfied, but not in the strong sense, presents itself as a limiting case in which the central axis meets $K(\Sigma)$ on the boundary.

We shall prove that in this limiting case a solution of the Signorini problem does not exist. This proves the necessity of the strong condition for the existence of a solution of the Signorini problem.

23 See [25, pp. 36-37].

24 If $K$ is a planar convex set and $x$ a point of $\partial K$, there exists a straight line $\lambda$ passing through $x$ such that $K$ lies in one of the two closed half-planes of origin $\lambda$.

Let us exclude the trivial case $K=\{x\}$. Consider a system of polar coordinates with pole $x$. If there existed two numbers $\alpha, \beta$ such that $\beta-\alpha \geqq \pi$ and such that for $\alpha<\theta<\beta$, the half axis originating in $x$ of argument $\theta$ did not contain any point of $K$ different from $x_{2}$ the assertion would be proved. Assume these numbers do not exist. Then there will be three values $\theta_{1}, \theta_{2}, \theta_{3}$ for which $0<\theta_{2}-\theta_{1}<\pi, 0<\theta_{3}-\theta_{2}<\pi, 0<\theta_{1}+2 \pi-\theta_{3}<\pi$ and such that the three half axes, determined by them, each contains a point of $K$ different from $x$. Let $x^{1}, x^{2}, x^{3}$ be these three points. The triangle $x^{1} x^{2} x^{3}$ is contained in $K$ and thus $x$ cannot be a point of $\partial K$.

Handbuch der Physik, Bd. VI a/2. 
10.IV If (10.1) is satisfied, but if the central axis of the system of applied forces meets $K(\Sigma)$ at a point of its boundary, then $\mathscr{I}(v)$ has no minimum in $V$.

Let $\varrho_{1}^{0}=a_{1}^{0}+b_{2}^{0} x_{3}-b_{3} x_{2}, \varrho_{2}^{0}=a_{2}^{0}+b_{3}^{0} x_{1}-b_{1}^{0} x_{3}, \varrho_{3}^{0}=a_{3}^{0}+b_{1}^{0} x_{2}-b_{2}^{0} x_{1}$ be a displacement of $R^{\prime}$ which is unilateral and such that

$$
\int_{A} f_{i} \varrho_{i}^{0} d x+\int_{\Sigma^{*}} g_{i} \varrho_{i}^{0} d \sigma=0
$$

Let $\lambda$ be the straight line of the plane $x_{3}=0$ given by the equation $\varrho_{3}^{0}=0$. Then, recalling (10.9), $\left(x_{\mathbf{1}}^{\mathbf{0}}, x_{\mathbf{2}}^{\mathbf{0}}\right) \in \lambda$. Note that the intersection of $\lambda$ with $K(\Sigma)$ is not empty.

Suppose there exists a solution $u$ of the Signorini problem. Then also $u^{0}=u+\varrho^{0}$ will be a solution of the problem (see Theorem 7.I). Since $\varrho_{3}^{\mathbf{0}}>0$ in $\Sigma-(\lambda \cap \partial \Sigma)$, we have $u_{3}^{0}>0$ a.e. in $\Sigma-(\lambda \cap \partial \Sigma)$. Let $z$ be a point of $\Sigma-(\lambda \cap \partial \Sigma)$ and let $D_{\delta}(z)$ be a 3-dimensional ball of center $z$ and radius $\delta$ at positive distance from $\lambda \cap \partial \Sigma$. Let $w \in \check{C}^{1}\left[D_{\delta}(z)\right]$. We can determine a positive number $t(\delta, z)$ such that for $|t|<t(\delta, z)$ the function $u^{0}+t w$ belongs to $V$. It follows that $I\left(u^{0}+t w\right)$ has a minimum for $t=0$. Then $B(u, w)-F(w)=0$. This implies that the measure $\gamma$ defined by Theorem 10.II is such that $\gamma(B)=0$ for each Borel set $B$ of $\Sigma$ contained in $D_{\delta}(z)$. It follows that the measure $\gamma$ is singular and the singular set of $\gamma$ must be contained in $\lambda \cap \partial \Sigma$.

Observe that $\gamma$ cannot be identically null, since, from (10.6), assuming $v_{1}=0$, $v_{2}=0, v_{3}=1$, we deduce

$$
\gamma(\lambda \cap \partial \Sigma)=-F_{3}(1)>0 .
$$

Thus: if there exists a solution of the Signorini problem, the reaction of the constraint is concentrated on the linear set $\lambda \cap \partial \Sigma$.

For every $v \in H_{1}(A) \cap C^{0}(A \cup \Sigma)$ we put

$$
\mathscr{I}_{0}(v)=\frac{1}{2} B(v, v)-F(v)-\int_{\lambda} v_{\partial \Sigma} d \gamma .
$$

Since we have $B(u, u)=F(u)$ and, because of (10.6),

we get

$$
\int_{\lambda \cap \delta \Gamma} v_{3} d \gamma=B(u, v)-F(v),
$$

$$
\mathscr{I}_{0}(v)-\mathscr{I}(u) \geqq B(u-v, u-v) \geqq 0 \text {. }
$$

Let $p$ be a positive constant greater than the diameter of $A$. We put

$$
v_{1}^{\delta} \equiv 0, \quad v_{2}^{\delta} \equiv 0, \quad v_{3}^{\delta}=\log \log \frac{\left(\left|a^{0}\right|+\left|b^{0}\right| p\right)^{2}+p^{2}+1}{\left|\varrho_{3}^{0}(x)\right|^{2}+x_{3}^{2}+\delta} .
$$

For $0<\delta<1$ we have $v^{\delta} \in C^{\infty}(\bar{A})$. On the other hand we have

$$
\lim _{\delta \rightarrow 0} \mathscr{I}_{0}\left[v^{\delta}\right]=-\infty, \quad \mathscr{I}_{0}\left[v^{\delta}\right] \geqq \mathscr{I}(u) .
$$

This proves that no function $u$ such as to minimize $\mathscr{I}(v)$ in $V$ exists.

11. Historical and bibliographical remarks concerning Existence Theorems in Elasticity. Existence theorems are of prominent interest in problems of mechanics and physics, since they provide a rational tool for proving, independently of any physical plausibility and experimental evidence, the consistency of a theory which brings into a mathematical scheme facts and phenomena of the physical world. Unfortunately, they very often constitute the most difficult part of the theory. 
Concerning the classical linear elasticity of homogeneous isotropic bodies, the first existence theorem was given for the 1st Boundary Value Problem by FREDHOLM $[8]^{25}$ as an application of the discovery of his fundamental theorems concerning integral equations. The same problem was also considered, always using the Fredholm integral equations, by LAURICella [21], Marcolongo [24] and, later, by Lichtenstein [23].

The use of integral equations in the 1st Boundary Value Problem (Dirichlet's Problem) is possible by considering the classical differential system of elasticity as homogeneous, with non-homogeneous the boundary values and by representing the solution as a "potential of a double layer" relative to the fundamental solution matrix, which was given for the differential system in question by Lord Kelvin [14] and by Somigliana [40].

The approach exactly parallels the one which is used in potential theory for getting the existence theorem for the Dirichlet problem relative to harmonic functions [13], [12]. Real difficulties arise when one tries to use the same method for the 2nd Boundary Value Problem of classical elasticity. Following the analogy with harmonic functions one would represent the solution as a "potential of a simple layer", just as is done in the 2nd Boundary Value Problem for harmonic functions (Neumann's Problem) [13], [12].

Unfortunately the system of integral equations which one gets is not a Fredholm system. In fact the "kernels" of these integral equations are not absolutely integrable, and the corresponding integrals have a meaning only if they are interpreted as "Cauchy singular integrals".

This has misled some authors, who have taken them to be Fredholm integral equations. Let us spend a few words on this phenomenon.

Let us consider the differential operator of classical elasticity in the case $r=3$, which we write as follows (see E.T.E., Sect. 12).

$$
L_{i} u=u_{i / k k}+\sigma u_{k / k i}
$$

where $\sigma$ is a constant $>\frac{1}{3}$. To this operator we associate the boundary operator $t u$ which represents the forces on the boundary, corresponding to the displacement $u$ :

$$
t_{i} u=u_{i / k} v_{k}+\sigma u_{k / k} \nu_{i}+\left(u_{k / i} \nu_{k}-u_{k / k} v_{i}\right) .
$$

In the bounded regular domain $A$ the Betti reciprocity theorem holds:

$$
\int_{\partial A}\left(u_{i} t_{i} v-v_{i} t_{i} u\right) d \sigma+\int_{A}\left(u_{i} L_{i} v-v_{i} L_{i} u\right) d x=0,
$$

which is analogous to the Green formula for the Laplace operator

$$
\int_{\partial A}\left(u \cdot \frac{\partial v}{\partial v}-v \frac{\partial u}{\partial v}\right) d \sigma+\int_{A}\left(u \Delta_{\mathbf{2}} v-v \Delta_{\mathbf{2}} u\right) d x=0 .
$$

This has induced several researchers to believe that the operator $t u$ plays in elasticity the same role as the operator $\partial u / \partial v$ plays in potential theory. But it must be remarked that if we consider instead of $t u$, the more general operator $t(u ; \lambda)$

$$
t_{i}(u ; \lambda)=u_{i / k} v_{k}+\sigma u_{k / k} v_{i}+\lambda\left(u_{k / i} v_{k}-u_{k / k} v_{i}\right),
$$

where $\lambda$ is an arbitrary constant, the reciprocity relation (11.1) still holds

$$
\int_{\partial A}\left[u_{i} t_{i}(v ; \lambda)-v_{i} t_{i}(u ; \lambda)\right] d \sigma+\int_{A}\left(u_{i} L_{i} v-v_{i} L_{i} u\right) d x=0 .
$$

Then $t(u ; \lambda)$ has the same right as $t u$ to play in elasticity the role of the normal derivative in potential theory. With respect to the theory of Fredholm integral equations, the operator $t(u ; \lambda)$ which behaves like the normal derivative in potential theory, is one that, $\partial A$ being supposed a Liapounov boundary (i.e. with a Hölder continuous normal field), when it operates on the Somigliana fundamental matrix

$$
\left\{\frac{1}{x-y}-\delta_{i j} \frac{\sigma}{2(1+\sigma)} \frac{\partial^{2}|x-y|}{\partial x_{i} \partial x_{j}}\right\} \quad(i, j=1,2,3),
$$

produces kernels $k_{i j}(x, y)$ which have a Fredholm singularity, i.e. are such that $k_{i j}(x, y)=$ $\mathscr{O}\left(|x-y|^{\alpha-2}\right) 0<\alpha \stackrel{1}{\leqq}$. It is not difficult to see that this happens if and only if $\lambda=\sigma(2+\sigma)^{-1}$.

${ }^{25}$ Numbers in boldface square brackets refer to the Bibliography at the end of E.T.E. Numbers in lightface square brackets refer to the Bibliography at the end of the present paper. 
Unfortunately the case concerning elasticity is $\lambda=1$. It follows that the Fredholm method can be applied to the 2nd Boundary Value Problem provided one considers as boundary operator $t\left[u ; \sigma(2+\sigma)^{-1}\right]$ which has no physical meaning. Actually this was done by LAURICELLA [21], who called $t\left[u ; \sigma(2+\sigma)^{-1}\right]$ the pseudo-tensions operator.

The real 2nd Boundary Value Problem of elasticity was studied by KoRN [18] in a very long paper by a very complicated method which uses integral equations and for the first time (see also [19]) introduces the inequalities, nowadays known as Korn's inequalities (see E.T.E., Sect. 12). FrIEDRICHS [10], citing the work of KoRN writes: "The author of the present paper has been unable to verify KorN's proof for the second case". Bernstein and ToupIN in their paper on KoRN's inequalities, after quoting FrIEDRICHS' statement, write: "With him we confess unability to follow KoRN's original treatment".

A few years later H. WeyL [35] tried to study the 2nd Boundary Value Problem of elasticity by using Fredholm integral equations obtained by means of the so-called antenna potential. However a certain hypothesis, which he assumes for carrying out his approach, has not been proved to hold in general.

It must be remarked that, in any case, H. WEYL's paper is of fundamental interest in elasticity for the analysis of the asymptotic distribution of eigenvalues in problems of elasticity.

For long time the theory of Boundary Value Problems in elasticity made no substantial progress in the case when $r>2$. On the other hand, in the case when $r=2$, many important achievements were obtained, mainly by Muskhelishvili, I. N. VeKuA and the Georgian school, using complex methods and the theory of singular integral equations on a curve (for extensive bibliography see [34]).

In 1947 FRIEDRICHS published an important paper [10] on $\gamma$-dimensional problems of elasticity. He gives the first acceptable proof of KorN's second inequality (the proof of the first one is almost trivial) and new proofs of the existence theorems for the 1st and the 2nd Boundary Value Problems of classical elasticity and for the related eigenvalue problems. His method is founded on the variational approach (by the same method FRIEDRICHS had given in 1928 the existence theorem for a clamped plate [9]) and he succeeds in proving, by employing his technique of "mollifiers", the interior regularity of the solutions.

In 1950 appeared paper [3] in which, by use of methods of functional analysis, new proofs of the existence theorems for the 1st and the 2nd Boundary Value Problems of elasticity were given and, for the first time, the existence theorem for the 3rd B.V.P. (mixed B.V.P.) was obtained. It is worthwhile to recall here briefly the method used in [3] for the proof of this theorem. We shall consider, for simplicity, the case of the Laplace operator. We shall also introduce some simplifications and shall use language more modern than that of [3].

Let $A$ be a bounded domain of $X^{3}$ with a Liapounov boundary $\partial A$, which is decomposed into two open hypersurfaces $\Sigma_{1}$ and $\Sigma_{2}$ which have a common border $\partial \Sigma_{1}=\partial \Sigma_{2}$ and no other point in common. We shall consider $\Sigma_{i}(i=1,2)$ as an open set respect to $\partial A$. Let us suppose that there exists a domain $A^{\prime}$ with a Liapounov boundary $\partial A^{\prime}$ and such that $A^{\prime}>A$, $\partial A^{\prime} \cap \partial A=\bar{\Sigma}_{1}$. Let $H_{\Sigma}$, be the subspace of $H_{1}(A)$ obtained as closure of the linear manifold of all the real valued functions $v$ such that $v \in C^{1}(A \overline{)})$, spt $v \cap \overline{\Sigma_{1}}=\phi$. Let $\delta$ be a function Hölder continuous on $\bar{\Sigma}_{\mathbf{2}}$. We wish to prove that there exists one and only one function $u$ such that

$$
u \in H_{\Sigma_{1}} \cap C^{2}(A) \cap C^{0}\left(\bar{A}-\partial \Sigma_{1}\right) \cap C^{1}\left(A \cup \Sigma_{2}\right) ; \quad \Delta_{2} u=0 \quad \text { in } A, \quad \frac{\partial u}{\partial \nu}=\delta \text { on } \Sigma_{2} .
$$

Let $\tilde{u}$ be a function such that $\tilde{u} \in C^{2}(A) \cap C^{1}(\bar{A}), \Delta_{2} \tilde{u}=0$ in $A, \tilde{u}=\delta$ on $\Sigma_{2}$. Such a function is easily obtained by suitably continuing $\delta$ on $\partial A$ and solving the corresponding Neumann problem in $A$. Let us introduce in $H_{1}(A)$ the new scalar product

$$
((u, v))=\int_{A} u_{/ i} v_{/ i} d x
$$

and, identifying two functions of $H_{1}(A)$ which differ by a constant, let us denote by $\mathscr{H}$ the corresponding Hilbert space. Considering $H_{\Sigma_{1}}$ as a subspace of $\mathscr{H}$, let $u$ be the orthogonal projection of $\tilde{u}$ on $H_{\Sigma_{1}}$. We have for any $v \in H_{\Sigma_{1}}$

$$
\int_{A} u_{/ i} v_{/ i} d x=\int_{A} \tilde{u}_{/ i} v_{/ i} d x=-\int_{\Sigma_{2}} v \delta d \sigma
$$

If we take any $\varphi \in \stackrel{\circ}{C}^{\infty}\left(A^{\prime}\right)$ and put

$$
v(x)=\int_{A^{\prime}} G(x, y) \varphi(y) d y,
$$


where $G(x, y)$ is the Green function of the Dirichlet problem for $\Delta_{2}$, relative to $A^{\prime}$, we have

$$
-\int_{\Sigma_{\mathbf{1}}} v \delta d \sigma=\int_{\boldsymbol{A}} u_{/ i} v_{/ i} d x=-\int_{\boldsymbol{\Sigma}_{\mathbf{z}}} u \frac{\partial v}{\partial v} d \sigma-\int_{\boldsymbol{A}} u \varphi d x .
$$

By the arbitrariness of $\varphi$ we easily deduce that

$$
\theta(y) u(y)=\int_{\Sigma_{2}} \delta(x) G(x, y) d \sigma_{x}-\int_{\Sigma_{2}} u(x) \frac{\partial}{\partial v_{x}} G(x, y) d \sigma_{x}
$$

with $\theta(y)=1$ if $y \in A, \theta(y)=0$ if $y \in A^{\prime}-\bar{A}$. From (11.4) it is easy to deduce, using standard arguments of potential theory (jump relations) that $u$ is a solution of the problem. If $u^{0}$ is another solution of the problem, since, for any $v \in H_{\Sigma_{1}}$, we have

from (11.2) we see that $u^{0} \equiv u .^{26}$

$$
\int_{A} u_{l_{i}}^{0} v_{i i} d x=-\int_{\Sigma_{2}} v \delta d \sigma
$$

This approach, since only with difficulty could it be extended to higher order elliptic systems with variable coefficients, was not followed in E.T.E. Nevertheless, over the approach used there the earlier one has the advantage of not requiring such severe restrictions upon $\partial A$.

One year after the publication of [3] a note of EIDUs [7] appeared on the mixed problem of elasticity. It must be remarked that Soviet mathematicians have been very active in the field of the existence theory for classical elasticity. Besides the above cited contributions of the Georgian school, let us quote here the papers of S. L. Sobolev [39], Michlin [31], S. Y. Kogan [15], E. N. Nikolsky [35] on the extension to elasticity of the Schwarz alternating method and, in particular, the work of Michlin and Kupradze. The former has considered elasticity problems from several points of view. In his monograph [32] he considers, besides the above quoted alternating method, the variational approach and he reviews the results obtained in this field by Soviet mathematicians. However he does not seem to be aware of some of the work done in the western world. In the monograph [25] MichLIN applies his theory of multidimensional singular integral equations to problems of elasticity. This theory, initiated by Tricomi [43] and GIRAUD [10], has been concluded by Michlin and is the starting point of the modern theory of pseudo-differential operators. MichLIN in [25] is able to solve the system of singular integral equations, to which the 2nd B.V.P. of classical elasticity gives rise when the solution is represented by simple layer potentials. Similar results were obtained by KUPRADZE [20], almost at the same time. He also uses Michlin's theory to solve the same system of singular integral equations.

Let us mention here, besides other relevant contributions of KUPRADze, his work on dynamic problems and on problems for heterogeneous media [20], [15-22].

The equilibrium problem for a heterogeneous elastic medium (an elastic body composed by two homogeneous isotropic bodies with different Lamè constants) was first posed by

${ }^{26}$ Let us remark that the use of the domain $A^{\prime}$ and of the corresponding Green's function $G(x, y)$, according to the procedure used in [3] (which was followed by KuPRADzE [20] for the analysis of mixed problems) can be avoided and a further simplification in the proof of the existence theorem introduced. To this end, instead of using $v(x)$ as given by (11.3), let us assume

where

$$
v(x)=\psi(x) \int_{A} s(x, y) \varphi(y) d y
$$

$$
s(x, y)= \begin{cases}(2 \pi)^{-1} \log |x-y| & \text { for } r=2, \\ {\left[(2-r) \omega_{r}\right]^{-1}|x-y|^{2-r}} & \text { for } r>2\end{cases}
$$

$\left(\omega_{r}\right.$ is the hypersurface measure of the unit sphere of $\left.X^{r}\right), \varphi(x) \in C^{\infty}$ and $\psi(x)$ is a $C^{\infty}$ function such that, given arbitrarily $x^{0}$ disjoint from $\overline{\Sigma_{1}}, \psi(x) \equiv 1$ for $\left|x-x^{0}\right| \leqq \varepsilon, \psi(x) \equiv 0$ for $\left|x-x^{0}\right| \geqq 2 \varepsilon ; \varepsilon$ is a positive number such that the ball $\bar{\Gamma}_{2 \varepsilon}:\left|x-x^{0}\right| \leqq 2 \varepsilon$ is disjoint from $\overline{\Sigma_{1}}$. It is not difficult to see that for $y \in \Gamma_{\varepsilon}$ one gets the representation

$$
\theta(y) u(y)=\int_{\Sigma_{\mathbf{2}}} \int_{\Gamma_{\boldsymbol{\varepsilon}}} \delta(x) s(x, y) d \sigma_{x}-\int_{\Sigma_{\mathbf{2}}} \underset{\Gamma_{\boldsymbol{\varepsilon}}}{ } u(x) \frac{\partial}{\partial \nu_{x}} s(x, y) d \sigma_{x}+h(y),
$$

where $h(y)$ is a harmonic function in $\Gamma_{\varepsilon}$. By using (11.5) in place of (11.4) one gets the same conclusions. 
Picone [30], who proposed a method for numerical solution. Papers by Lions [28] and by Campanato [2] are concerned with this problem. The case of anisotropic bodies, with general elasticities, is for the first time considered in E.T.E. In that article the modern approach to boundary value problems for strongly elliptic operators is followed, which has made it possible, for the first time, to treat with great generality dynamic problems as well as diffusion problems and integro-differential problems. There can be no doubt that the analytical investigations of problems of elasticity have greatly contributed to the modern development of the theory of partial differential equations. For instance researches concerning the Korn inequalities are among the first examples of investigations connected with the concept of "coerciveness" now very important. In this respect, besides the papers of FRIEDRICHS and of BERNSTEIN and Toupin, already cited, let us mention here the work of Campanato [3], [4], which is mainly interesting for the examples in which Korn's inequalities fail, and a remarkable paper by PAYNE and Weinberger [29], where the best estimate for the 2nd Korn inequality, in the case of a sphere, is obtained and a new proof of this inequality provided for a class of domains. Unfortunately the results of the paper do not have so large a range of validity as the authors claim. The paper [13] of GoBERT also concerns Korn's inequality.

The first author to consider a unilateral problem for elasticity was SignORINI [37] early in 1933. He presented again his theory, in more complete fashion, in 1959 [38]. In this paper the problem nowadays known as the Signorini problem is proposed. This problem was investigated and solved in the paper [6], which was submitted for publication in September, 1963, and which appeared in 1964. The results of paper [6] had been announced in [8] (February 1963), in [5] and in [9]. The results of [6], although relative to a specific problem of elasticity, are immediately extensible - as far as the abstract theory is concerned - to an abstract unilateral problem relative to a symmetric semi-coercive bilinear form considered in a cone.

The existence and uniqueness theorem given in [6] is the first example of an existence and uniqueness theorem for a unilateral problem connected with a differential operator.

More than one year later Stampacchia [41] considered unilateral problems relative to nonsymmetric coercive bilinear forms. The case considered by Stampacchia because of the coerciveness hypothesis he assumes cannot cover that considered in [6]. In fact-as has been shown in the present paper-it is just the absence of the coerciveness condition which makes the problem a complicated one, since, in this case, one has to face the delicate question of the compatibility conditions. When the bilinear form is coercive, the problem is almost trivial in the symmetric case. The non symmetric case is easily reduced to the symmetric one by a simple argument, shown in [30], which makes use of a suitable contraction mapping. We have used this argument at the beginning of the proof of Theorem 2.I of the present paper. The authors of [30] consider also the case of non-coercive non-symmetric bilinear forms. In the abstract scheme which they assume, they exactly reproduce the situation which arises in [6] in connection with elasticity and give a theorem which imitates the results of [6], transferring them into their abstract setting. The proof of the boundedness of the sequence which furnishes the solution of their unilateral problem is strongly inspired by the proof of the boundedness of the analogous sequence, presented in [6]. Unfortunately, imitation of the results of [6] (which relate to a particular convex set) in the case of the general convex set which they introduce, without considering the extremely more general geometric nature of an arbitrary convex set, lead them to state unacceptable results. As a matter of fact, if one uses results of [30] for solving unilateral problems for non-coercive forms, one has to impose compatibility conditions on the data, even when that is not necessary.

Nowadays the literature on unilateral problems grows more and more. ${ }^{27}$ The abstract point of view has especially considered extensions to non linear operators. These researches mainly interest pure mathematics. However, a number of concrete specific problems have also been investigated and the associated questions of regularization studied. While I believe that the abstract theory, developed in this paper, is able to cover with sufficient generality the unilateral problems connected with linear operators of applied mathematics, I realize that the regularization theory is still very far from the generality that it has in the case of bilateral problems. The regularization results stated in the present paper, as far as the relevant differential operator is concerned, are the most general known up to date for linear operators.

With specific reference to elasticity, let us mention that one of the first unilateral problems in elasticity after SignorinI's was formulated by PRAGER [36]. It has been considered in Sect. 6 (example V) of the present paper. A unilateral problem for a membrane was investigated by H. Lewy [26] and later by Lewy and Stampacchia [27]. Elastic plastic torsion problems have been studied by T. W. TING [42], Duvaut and Lanchon [24] and LANCHON [23]. The theory of elastic-plastic torsion problem is explained in detail in the

27 For a bibliography on this subject we refer to the book [29]. 
article by TING in volume VIa/3 of this Encyclopedia. Duvaut has considered the Signorini problem in visco-elasticity [5] (i.e. hereditary elasticity); Grioli [11] and Duvaut [6] elastic problems when the bounding surface has friction. The paper [33] of MoREAU concerns unilateral problems of elastostatics; he has considered also a number of abstract and concrete topics connected with unilateral problems.

So far we have considered problems connected with linear elasticity. Although the unilateral problems do not belong to the domain of linear analysis and many questions are still not settled, it has been possible to put them in a general scheme. That is not possible for nonlinear elasticity. As far as existence theory is concerned, there are particular results, for special problems but nothing sufficient to give concrete foundations to a general theory. That probably will be one of the tasks of applied analysts in the next years.

\section{Bibliography.}

[1] Bernstein, B., and R. A. Toupin: Korn inequalities for the sphere and circle. Arch. Rational Mech. Anal. 6 (1960).

[2] Campanato, S.: Sul problema di M. Picone relative all'equilibrio di un corpo elastico incastrato. Ricerche di Matem. VI (1957).

[3] - Sui problemi al contorno per sistemi di equazioni differenziali lineari del tipo dell'elasticità. Parte I and Parte II. Ann. Scuola Norm. Sup. Pisa, s. III, 13 (1959).

[4] - Proprietà di taluni spazi di Banach connessi con la teoria dell'elasticità. Ann. Scuola Norm. Sup. Pisa, s. III, 16 (1962).

[5] Duvaut, G.: Problème de Signorini en viscoélasticité linéaire. Compt. Rend. 268 (1969).

[6] - Problèmes unilatéraux en mécanique des milieux continus. Proceedings of the International Congress of Mathematicians, Nice (1970).

[7] Eidus, D. M.: On a mixed problem of elasticity theory [in Russian]. Dokl. Akad. Nauk USSR 76 (1971).

[8] Fichera, G.: Sul problema elastostatico di Signorini con ambigue condizioni al contorno. Rend. Accad. Naz. Lincei, s. VIII, 34 (1963).

[9] - Un teorema generale di semicontinuità per gli integrali multipli e sue applicazioni alla fisica-matematica. Atti del Convegno Lagrangiano, Acc. Sci. Torino (1963).

[10] Giraud, G.: Equations à intégrales principales. Ann. Sci. École Norm. Supér. 51 (1934).

[11] Grioli, G.: Problemi d'integrazione e formulazione integrale del problema fondamentale dell'elastostatica. Simposio intern. sulle Applicazioni della Analisi alla Fisica Matematica, Cagliari-Sassari (1964). Roma: Edit. Cremonese 1965.

[12] GüNTER, N. M.: Die Potentialtheorie und ihre Anwendung auf Grundaufgaben der Mathematischen Physik. Leipzig: B. G. Teubner 1957.

[13] KellogG, O. D.: Foundations of potential theory. Berlin: Springer 1929; New York: F. Ungar 1946.

[14] Kelvin, Lord (W. Thomson): Note on the integration of the equations of equilibrium of an elastic solid. Cambridge \& Dublin Math. J. (1848).

[15] Kogan, S. Y.: On the resolution of the threedimensional problem of elasticity by means of the Schwarz alternating method [in Russian]. Izv. Akad. Nauk (Geophys. Ser.) 3 (1956).

[16] Kupradze, V. D.: Boundary value problems of the theory of forced elastic oscillations [in Russian]. Usp. Matem. Nauk 8 (1953).

[17] - Some new theorems on oscillation equations and their application to boundary value problems [in Russian]. Tr. Tbilisk. Universiteta 25a (1944).

[18] - Boundary value problems of the theory of elasticity for piecewise-homogeneous elastic bodies [in Russian]. Soobshch. Akad. Nauk. Gruz. 22 (1959).

[19] - On the theory of boundary value problems for inhomogeneous elastic bodies [in Russian]. Soobshch. Akad. Nauk Gruz. 22 (1959).

[20] - On boundary value problems of the theory of elasticity for piecewise-homogeneous bodies [in Russian]. Soobshch. Akad. Nauk Gruz. 22 (1959).

[21] - The boundary value problems of the oscillation theory and their integral equations [in Russian]. Moscow: Gostekhizdat (1950).

[22] - Dynamical problems in elasticity (from Progress in solid mechanics), vol. 3. Amsterdam: North Holland (1963).

[23] Lanchon, H.: Solution du problème de torsion élastoplastique d'une barre cylindrique de section quelconque. Compt. Rend. 269 (1969).

[24] -, et G. Duvaut: Sur la solution du problème de torsion élastoplastique d'une barre cylindrique de section quelconque. Compt. Rend. 264 (1967).

[25] Levi Civita, T., e U. Amaldi: Compendio di Meccanica razionale. II Ediz. N. Zanichelli (1938). 
[26] LEwy, H.: On a variational problem with inequalities on the boundary. J. Math. \& Mech. 17 (1968).

[27] - , and G. Stampacchia: On the regularity of the solution of a variational inequality. Comm. Pure Appl. Math. 22 (1969).

[28] Lions, J. L.: Contribution à un problème de M. Picone. Ann. Matem. pura e appl., s. IV, 16 (1955).

[29] - Quelques méthodes de résolution des problèmes aux limites non linéaires. Paris: Dunod Gauthier-Villars (1969).

[30] -, and G. Stampacchia: Variational inequalities. Comm. Pure Appl. Math. 20 (1967).

[31] Michlin, S. G.: On the Schwarz algorithm [in Russian]. Dokl. Akad. Nauk USSR 77 (1951).

[32] - The problem of the minimum of a quadratic functional. S. Francisco-London-Amsterdam: Holden-Day 1965.

[33] Moreau, J. J.: La notion de sur-potential et les liasons unilatérales en élastostatique. Proc. of the 12th Inter. Congress of Appl. Mech. Stanford (1968).

[34] Muskhelishvili, N. I.: Singular integral equation, 2nd ed. Groningen: Nordhoff 1959, and 3rd ed. [in Russian] published by the State Publ. House for Physics-Mathematics Literature, Moscow (1968).

[35] Nikolski, E. N.: The Schwarz algorithm in the problem of tensions in the theory of elasticity [in Russian]. Dokl. Akad. Nauk USSR 135 (1960).

[36] Prager, W.: Unilateral constraints in mechanics of continua. Atti del Convegno Lagrangiano, Acc. Sci. Torino (1963).

[37] Signorini, A.: Sopra alcune questioni di elastostatica. Atti Soc. Ital. per il Progesso delle Scienze (1933).

[38] - Questioni di elasticità non linearizzata o semi-linearizzata. Rend. di Matem. e delle sue appl. 18 (1959).

[39] Sobolev, S. L.: The Schwarz algorithm in elasticity theory. Dokl. Akad. Nauk USSR 4 (1936).

[40] Somigliana, C.: Sopra l'equilibrio di un corpo elastico isotropo. Nuovo Cimento 3 (1885).

[41] Stampacchia, G.: Formes bilinéaires coercitives sur les ensembles convexes. Compt. Rend. 258 (1964).

[42] TING, T. W.: Elastic-plastic torsion problem. II Arch. Rat. Mech. Anal. 25 (1967); III Arch. Rat. Mech. Anal. 34 (1969).

[43] TRIcomi, F.: Equazioni integrali contenenti il valor principale di un integrale doppio. Math. Z. 27 (1928). 